\title{
Hyperspectral super-resolution accounting for spectral variability: coupled tensor LL1-based recovery and blind unmixing of the unknown super-resolution image*
}

\author{
Clémence Prévost ${ }^{\dagger}$, Ricardo A. Borsoi ${ }^{\ddagger} \S$, Konstantin Usevich ${ }^{\dagger}$, David Brie ${ }^{\dagger}$, José C. M. \\ Bermudez $z^{\ddagger}$, and Cédric Richard ${ }^{\S}$
}

\begin{abstract}
In this paper, we propose to jointly solve the hyperspectral super-resolution problem and the unmixing problem of the underlying super-resolution image using a coupled LL1 block-tensor decomposition. We consider a spectral variability phenomenon occurring between the observed low-resolution images. Exact recovery conditions for the image and mixing factors are provided. We propose two algorithms: an unconstrained one and another one subject to non-negativity constraints, to solve the problems at hand. We showcase performance of the proposed approach on synthetic and real images.
\end{abstract}

Key words. Hyperspectral super-resolution, spectral variability, hyperspectral unmixing, image fusion, tensor decompositions.

AMS subject classifications. $68 \mathrm{U} 10,62 \mathrm{H} 35,94 \mathrm{~A} 08$

\section{Introduction.}

1.1. Background. Hyperspectral devices are able to sample the electromagnetic spectrum into hundred of wavelengths, allowing for the acquisition of hyperspectral images (HSIs) that possess high spectral resolution. However, the tradeoff between spatial and spectral resolution forces the HSIs to have a small number of relatively large pixels [41]. On the other hand, multispectral sensors produce multispectral images (MSIs) with high spatial resolution (smaller pixels), at the cost of a restricted number of spectral bands. The composition of each pixel in HSIs and MSIs can be approximated by a sum of a small number of spectral signatures, or endmembers. This representation is known as the linear mixing model, and allows for identification of the materials and their abundances in a scene, a process termed unmixing. Many unmixing approaches have been proposed (see [3, 37, 36] and references therein).

The hyperspectral super-resolution (HSR) problem [50] was formulated to circumvent the physical limitations of each device. This problem aims at recovering a super-resolution image (SRI) that possesses both high spatial and high spectral resolutions from co-registered HSI and MSI of the same scene. The high spatial and spectral resolutions of the SRI can then be exploited in unmixing tasks.

\footnotetext{
${ }^{\dagger}$ Centre de Recherche en Automatique de Nancy (CRAN), Université de Lorraine, CNRS, Vandoeuvre-lèsNancy, France.

${ }^{\ddagger}$ Department of Electrical Engineering, Federal University of Santa Catarina (DEE-UFSC), Florianópolis, SC, Brazil.

${ }^{\S}$ Université Côte d'Azur, Nice, France, Lagrange Laboratory (CNRS, OCA). e-mail: firstname.lastname@unice.fr.

*Submitted to the editors October 25, 2021.

Funding: This work was partly supported by the ANR (Agence Nationale de Recherche) under grant LeaFleT (ANR-19-CE23-0021), by the National Council for Scientific and Technological Development (CNPq) under grants 304250/2017-1, 409044/2018-0, 141271/2017-5 and 204991/2018-8. The work of C. Richard was supported through the UCA JEDI Investments in the Future project with the reference number ANR-15-IDEX-0001.

We also thank the GdR CNRS ISIS for supporting the collaboration between R. A. Borsoi and C. Prévost, K. Usevich and $\mathrm{D}$. Brie through a bourse de mobilité.
} 
Since there exist few satellites that carry both hyperspectral and multispectral sensors [15, 29], combining an HSI and an MSI acquired onboard of different missions has become a task of prime interest $[22,17]$. Since the HSI and the MSI are acquired at different time instants, their acquisition conditions can differ by, e.g., illumination, atmospheric or seasonal changes [5]. This can cause variations in the underlying endmembers and impact negatively the HSR and unmixing algorithms. This phenomenon is known as spectral variability.

In this paper, we propose to formulate the HSR problem as a coupled tensor block-term decomposition (BTD) of the HSI and MSI, accounting for spectral variability between the endmembers. Inspired by the works of $[54,53,13]$, we propose guarantees for noiseless exact recovery of the SRI and its latent factors based on the linear mixing model. One advantage of using the linear mixing model is that the factors of a properly chosen decomposition can be seen as the high-resolution spectral signatures and abundance maps corresponding to the materials in the underlying SRI, provided that they are entry-wise non-negative. Differently from matrix-based models, our noiseless recovery conditions do not require additional constraints on the low-rank factors. We also propose a unified procedure that aims at estimating the latent mixing factors and recovering the SRI in one single step. Our experiments illustrate the competitive performance of the proposed approach for HSR. The performance for unmixing of the unknown SRI is compared to that of traditional unmixing algorithms applied on an estimated SRI.

Many approaches have been proposed to solve the HSR problem. Most matrix approaches $[51,49,42,48]$ are based on the linear mixing model and perform a coupled low-rank factorization of the matricized HSI and MSI. Some matrix approaches provide exact recovery conditions for the SRI in noiseless cases. However, these recovery guarantees usually require additional priors on the model. See for example [33], which promotes sparsity of the factors. In the absence of such hypotheses, only a bound on the recovery error can be obtained [34]. Some matrix approaches are suitable for the HSR and unmixing problem as well, which consists of recovering the underlying SRI by means of a physically-informed low-rank approximation. See for instance [33] and [51]. However, identifiability of the mixing model could only be obtained under additional constraints on the low-rank factors [14, 32]. As a result, to the best of our understanding, recovery conditions for the joint HSR and unmixing problem in the literature only consider specific classes of problems.

More recently, tensor-based approaches were proposed for the HSR problem. The works of $[27,28]$ formulate the HSR problem as a coupled canonical polyadic (CP) decomposition, while a coupled multilinear Tucker decomposition is used in [38]. However, the factors of these decompositions lack physical interpretation, and thus the aforementioned methods cannot be used for unmixing. Motivated by the usefulness of tensor models, approaches based on blocktensor decomposition [53, 13] were proposed for solving the HSR problem. This decomposition was also successfully used to perform unmixing [39] on the SRI directly.

Most of these approaches however share a common limitation: they assume that the acquisition conditions of the HSI and MSI are the same, hence they ignore the variability phenomenon. In [4], a super-resolution method accounting for seasonal spectral variability was proposed. Using a low-rank matrix formulation, the spectral signatures underlying the HSI and MSI were allowed to be different from each other, with variations introduced by a set of multiplicative scaling factors [25]. This algorithm led to significant performance improvements when the HSI and MSI are subject to spatially uniform seasonal or acquisition variations. However, the algorithm in [4] presented high computation times and did not offer any theoretical guarantees. In [6], two tensor-based algorithms based on the Tucker decom- 
position were proposed, accounting for spatially and spectrally-localized changes between the HSI and MSI. Noiseless unique recovery guarantees were proposed. Unfortunately, the Tucker decomposition being generally non-unique, the decomposition factors were not physically interpretable.

The paper is organized as follows. The remainder of Section 1 contains tensor algebra preliminaries. Section 2 introduces the low-rank tensor model, and the model for spectral variability. Section 3 addresses recovery analysis for the joint HSR and unmixing task. Section 4 describes the proposed algorithms and their computational complexity. Finally, Sections 5 and 6 contain numerical experiments for HSR and coupled unmixing, respectively.

1.2. Definitions and notations. We follow the notations of [31, 10]. We use lower $(a)$ or uppercase $(A)$ plain font for scalars, boldface lowercase $(\boldsymbol{a})$ for vectors, boldface uppercase $(\boldsymbol{A})$ for matrices and calligraphic $(\boldsymbol{A})$ for tensors. The elements of vectors, matrices and tensors are denoted as $a_{i}, A_{i, j}$ and $\mathcal{A}_{i_{1}, \ldots, i_{N}}$, respectively. The transpose of a matrix $\boldsymbol{A}$ is denoted by $\boldsymbol{A}^{\top}$. We use $\boldsymbol{I}_{N}$ for the $N \times N$ identity matrix and $\mathbf{0}_{L \times K}$ for the $L \times K$ matrix of zeros. Notation $\mathbb{1}_{L}$ denotes an all-ones vector of size $L \times 1$. For a matrix $\boldsymbol{X}$, the notation $\boldsymbol{X} \geq \mathbf{0}$ means that $\boldsymbol{X}$ is entry-wise non-negative. Symbols $\boldsymbol{\otimes}$ and $\odot$ denote the Kronecker and Khatri-Rao products, respectively. The Hadamard (element-wise) product is denoted by $\square$. We use vec $\{\cdot\}$ for the standard column-major vectorization of a matrix or a tensor. For two matrices $\boldsymbol{A}$ and $\boldsymbol{B}$, the operator $\operatorname{Diag}\{\boldsymbol{A}, \boldsymbol{B}\}$ produces a block-diagonal matrix whose diagonal blocks are $\boldsymbol{A}$ and $\boldsymbol{B}$. Each dimension of a tensor is called a mode, and the number of dimensions is called order. A mode- $p$ fiber of tensor $\mathcal{X}$ is a vector obtained from $\mathcal{X}$ by fixing all but the $p$-th dimension. A slab or slice of a tensor $\mathcal{X}$ is a matrix whose columns are the vectors of $\mathcal{X}$ obtained by fixing all but two of its modes. We restrict the scope of this paper to order-3 tensors.

Definition 1.1. Outer product - The outer product between three vectors $\boldsymbol{a} \in \mathbb{R}^{I}, \boldsymbol{b} \in \mathbb{R}^{J}$, $\boldsymbol{c} \in \mathbb{R}^{K}$ is an order-3 tensor of rank 1 defined as $\mathcal{X}=\boldsymbol{a} \otimes \boldsymbol{b} \otimes \boldsymbol{c} \in \mathbb{R}^{I \times J \times K}$. Each element of $\mathcal{X}$ is accessed as $\mathcal{X}_{i, j, k}=a_{i} b_{j} c_{k}$.

Definition 1.2. Tensor unfoldings - The mode-p unfolding of a tensor $\mathcal{X}$, denoted by $\boldsymbol{X}^{(p)}$, is the matrix whose rows are the mode- $p$ fibers of $\mathcal{X}$, ordered according to the vectorization order. For a third-order tensor $\mathcal{X} \in \mathbb{R}^{I \times J \times K}$, we have $\boldsymbol{X}^{(1)} \in \mathbb{R}^{J K \times I}, \boldsymbol{X}^{(2)} \in \mathbb{R}^{I K \times J}$ and $\boldsymbol{X}^{(3)} \in \mathbb{R}^{I J \times K}$.

Definition 1.3. Mode product - The mode-p product between a tensor $\mathcal{X}$ and a matrix $\boldsymbol{M}$ is denoted by $\mathcal{X} \bullet_{p} \boldsymbol{M}$ and is evaluated such that each mode-p fiber of $\mathcal{X}$ is multiplied by $\boldsymbol{M}$. For instance, the elements of the mode-1 product between $\mathcal{X} \in \mathbb{R}^{I \times J \times K}$ and $\boldsymbol{M} \in \mathbb{R}^{L \times I}$ are determined as $\left(\mathcal{X} \bullet_{1} \boldsymbol{M}\right)_{\ell, j, k}=\sum_{i} \mathcal{X}_{i, j, k} \boldsymbol{M}_{\ell, i}, \ell \in\{1, \ldots, L\}$. Moreover, it holds that $\mathcal{Y}=\mathcal{X} \bullet \bullet_{k} \boldsymbol{M} \Leftrightarrow \boldsymbol{Y}^{(k)}=\boldsymbol{X}^{(k)} \boldsymbol{M}^{\top}$.

1.3. Block-term decomposition with ranks $(L, L, 1)$. In this subsection, we introduce the block-term decomposition with ranks $(L, L, 1)$, that we will use to build our model. The main advantage of this decomposition is to link the low-rank factors to high-resolution abundance matrices and spectral signatures used inunmixing of the unknown SRI, under additional nonnegativity priors on the low-rank factors. We also recall some sufficient uniqueness conditions for this decomposition, as well as useful properties. 
Definition 1.4. Block-term decomposition - An order-3 tensor $\mathcal{X} \in \mathbb{R}^{I \times J \times K}$ generally admits a block-term decomposition (BTD) with ranks $(L, L, 1)$ (LL1-BTD) as

$$
\mathcal{X}=\sum_{r=1}^{R}\left(\boldsymbol{A}_{r} \boldsymbol{B}_{r}^{\top}\right) \otimes \boldsymbol{c}_{r}
$$

where $\boldsymbol{A}_{r} \in \mathbb{R}^{I \times L}, \boldsymbol{B}_{r} \in \mathbb{R}^{J \times L}$, and $\boldsymbol{c}_{r} \in \mathbb{R}^{K}$, for $r \in\{1, \ldots, R\}$. Moreover, we denote $\boldsymbol{A}=\left[\boldsymbol{A}_{1}, \ldots, \boldsymbol{A}_{R}\right] \in \mathbb{R}^{I \times L R}, \boldsymbol{B}=\left[\boldsymbol{B}_{1}, \ldots, \boldsymbol{B}_{R}\right] \in \mathbb{R}^{J \times L R}$ and $\boldsymbol{C}=\left[\boldsymbol{c}_{1}, \ldots, \boldsymbol{c}_{R}\right] \in \mathbb{R}^{K \times R}$.

Theorem 1.5. [12, Theorem 4.7] Let $(\boldsymbol{A}, \boldsymbol{B}, \boldsymbol{C})$ denote an LL1-BTD of a tensor $\mathcal{X}$ for $r \in\{1, \ldots, R\}$ as in (1.1). Assume that $(\boldsymbol{A}, \boldsymbol{B}, \boldsymbol{C})$ are drawn from certain joint absolutely continuous distributions. If $I J \geq L^{2} R$ and

$$
\min \left(\left\lfloor\frac{I}{L}\right\rfloor, R\right)+\min \left(\left\lfloor\frac{J}{L}\right\rfloor, R\right)+\min (K, R) \geq 2 R+2
$$

then $\boldsymbol{A}_{r} \boldsymbol{B}_{r}^{\top}$ and $\boldsymbol{c}_{r}$ are essentially unique almost surely for $r \in\{1, \ldots, R\}$.

Definition 1.6. Partition-wise Khatri-Rao product - The partition-wise Khatri-Rao product between two partitioned matrices $\boldsymbol{A}$ and $\boldsymbol{C}$ defined as above can be expressed as

$$
\boldsymbol{C} \odot_{p} \boldsymbol{A}=\left[\boldsymbol{c}_{1} \otimes \boldsymbol{A}_{1}, \ldots, \boldsymbol{c}_{R} \otimes \boldsymbol{A}_{R}\right] \in \mathbb{R}^{I K \times L R} .
$$

Property 1.7. Tensor unfoldings and LL1 - Using the above notations, the unfoldings of a tensor $\mathcal{X}$ admitting an LL1-BTD as above can be expressed as

$$
\begin{aligned}
& \boldsymbol{X}^{(1)}=\left(\boldsymbol{C} \odot_{p} \boldsymbol{B}\right) \boldsymbol{A}^{\top}, \\
& \boldsymbol{X}^{(2)}=\left(\boldsymbol{C} \odot_{p} \boldsymbol{A}\right) \boldsymbol{B}^{\top}, \\
& \boldsymbol{X}^{(3)}=\left[\left(\boldsymbol{A}_{1} \odot \boldsymbol{B}_{1}\right) \mathbb{1}_{L}, \ldots,\left(\boldsymbol{A}_{R} \odot \boldsymbol{B}_{R}\right) \mathbb{1}_{L}\right] \boldsymbol{C}^{\boldsymbol{\top}} .
\end{aligned}
$$

\section{Proposed model.}

2.1. Degradation model and indeterminacies. We consider an HSI data cube $\mathcal{Y}_{H} \in$ $\mathbb{R}^{I_{H} \times J_{H} \times K}$ and an MSI data cube $\mathcal{Y}_{M} \in \mathbb{R}^{I \times J \times K_{M}}$. The scalars $K$ and $K_{M}$ denote the spectral dimensions, and $(I, J)$ (resp. $\left.\left(I_{H}, J_{H}\right)\right)$ stand for the spatial dimensions. We suppose that the spatial resolution of the MSI is higher than that of the HSI (i.e., $I_{H}<I$ and $J_{H}<J$ ), while its spectral resolution is lower $\left(K_{M}<K\right)$. Most previous works [27]-[13] considered the low resolution images as degraded versions of a single SRI $\mathcal{Z} \in \mathbb{R}^{I \times J \times K}$, that possesses high spatial and spectral resolutions. This model can be expressed as:

$$
\left\{\begin{array}{l}
\mathcal{Y}_{H}=\mathcal{Z} \bullet \bullet_{1} \boldsymbol{P}_{1} \bullet_{2} \boldsymbol{P}_{2}+\mathcal{E}_{H} \\
\mathcal{Y}_{M}=\mathcal{Z} \bullet \boldsymbol{P}_{3}+\mathcal{E}_{M}
\end{array}\right.
$$

where the tensors $\mathcal{E}_{H}$ and $\mathcal{E}_{M}$ are additive noise terms. The matrix $\boldsymbol{P}_{3} \in \mathbb{R}^{K_{M} \times K}$ contains the spectral response functions for each band of the MSI sensor. The spatial degradation matrices $\boldsymbol{P}_{1} \in \mathbb{R}^{I_{H} \times I}$ and $\boldsymbol{P}_{2} \in \mathbb{R}^{J_{H} \times J}$ perform Gaussian blurring and downsampling along each spatial dimension, i.e. we suppose that the spatial degradation operation is separable, as in the commonly used Wald's protocol [47]. 
However, this model implies that the acquisition conditions of $\mathcal{Y}_{H}$ and $\mathcal{Y}_{M}$ are the same, thus it ignores possible variations in atmospheric, seasonal or illumination conditions $[44,52]$ between the HSI and MSI. The variability phenomenon motivates the need for more flexible models. As a result, in this paper, we adopt a more general approach. As in [6], we consider two different SRIs $\mathcal{Z} \in \mathbb{R}^{I \times J \times K}$ and $\widetilde{\mathcal{Z}} \in \mathbb{R}^{I \times J \times K}$, underlying the HSI and MSI, respectively. The SRIs $\mathcal{Z}$ and $\widetilde{\mathcal{Z}}$ contain possibly different spectral signatures and can be linked as

$$
\widetilde{\mathcal{Z}}=\mathcal{Z}+\Psi
$$

where $\boldsymbol{\Psi} \in \mathbb{R}^{I \times J \times K}$ is a tensor of spectral variability. This leads to the following model:

$$
\left\{\begin{array}{l}
\mathcal{Y}_{H}=\tilde{\mathcal{Z}} \bullet_{1} \boldsymbol{P}_{1} \bullet_{2} \boldsymbol{P}_{2}+\mathcal{E}_{H}, \\
\mathcal{Y}_{M}=\widetilde{\mathcal{Z}} \bullet_{3} \boldsymbol{P}_{3}+\mathcal{E}_{M}
\end{array}\right.
$$

In this framework, the HSR problem consists recovering $\mathcal{Z} \in \mathbb{R}^{I \times J \times K}$ and $\boldsymbol{\Psi} \in \mathbb{R}^{I \times J \times K}$ under the assumption of the observation model (2.2)-(2.3). However, the presence of the variability tensor $\boldsymbol{\Psi}$ makes this problem ambiguous [6], as one cannot easily separate $\mathcal{Z}$ and $\Psi$ from $\widetilde{\mathcal{Z}}$. We recall the following theorem [6]:

Theorem 2.1. [6, Theorem 1.a)] Suppose that the HSI and MSI are generated according to (2.3) and that the observation noise is zero (i.e. $\mathcal{E}_{H}, \mathcal{E}_{M}=\mathbf{0}$ ). If either $\boldsymbol{P}_{1}, \boldsymbol{P}_{2}$ or $\boldsymbol{P}_{3}$ have non-trivial nullspace, then $(\mathcal{Z}, \Psi)$ cannot be uniquely recovered from $\mathcal{Y}_{H}$ and $\mathcal{Y}_{M}$.

In [6], approaches based on model (2.3) were proposed, using a coupled Tucker approximation. However, due to the non-uniqueness of the Tucker decomposition, the latent multilinear factors were not guaranteed to be unique, and no non-negativity constraints were enforced. Thus it was not possible to incorporate them into an interpretable mixing model. In what follows, we introduce a low-rank tensor model to circumvent the fundamental ambiguities of the proposed degradation model. Indeed, a wisely chosen low-rank decomposition might still allow for unique recovery of portions of the tensors. We propose to use the LL1-BTD, whose factors are suitable for physical interpretation. The LL1-BTD model was successfully used for unmixing [39] and HSR [53, 13]. However, these works ignored any variability phenomenon.

Differently from [6] (that considered spatially and spectrally localized changes), we consider that variability only impacts the spectral dimension of the SRI. This is reasonable, since spectral variability can occur even with short acquisition time differences. This assumption also allows for sometimes less restrictive noiseless recoverability guarantees than the ones from $[6]^{*}$. As a result, the proposed model is more suitable for scenarios with low spatial variability. Nevertheless, we will show in Sections 5 and 6 that it is able to address large acquisition time differences as well.

2.2. LL1-BTD mixing model for the underlying SRIs. In the linear mixing model, each pixel of the SRI $\mathcal{Z}$ (and therefore, of the HSI $\mathcal{Y}_{H}$ ) can be represented as a sum of a small number $R$ of pure spectral signatures [30]. This property can be incorporated in a physicallyinformed low-rank approximation model, allowing to perform both HSR and unmixing in a unified procedure. Thus, as in [53], we can model the third-mode unfolding of $\mathcal{Z}$ as:

$$
\boldsymbol{Z}^{(3)}=\boldsymbol{S C}^{\boldsymbol{\top}} \in \mathbb{R}^{I J \times K},
$$

\footnotetext{
${ }^{*}$ because of the more general variability model.
} 
where $\boldsymbol{C}=\left[\boldsymbol{c}_{1}, \ldots, \boldsymbol{c}_{R}\right] \in \mathbb{R}^{K \times R}$ is a matrix containing the spectral signatures of the $R$ endmembers underlying the SRI. The matrix $\boldsymbol{S}=\left[\operatorname{vec}\left\{\boldsymbol{S}_{1}\right\}, \ldots, \operatorname{vec}\left\{\boldsymbol{S}_{R}\right\}\right] \in \mathbb{R}^{I J \times R}$ contains the vectorized abundance maps of each material. In fact, (2.4) can be viewed as the linear mixing model for the SRI $\mathcal{Z}$, under the assumption that $\boldsymbol{C}$ and $\boldsymbol{S}$ are entry-wise non-negative ${ }^{\dagger}$.

We assume as in [53] that the abundance maps admit low rank $L$. The hypothesis of lowrank abundance matrices is reasonable, since the two spatial dimensions are often correlated along the rows and columns, respectively. Thus we have

$$
\boldsymbol{S}_{r} \approx \boldsymbol{A}_{r} \boldsymbol{B}_{r}^{\top} \in \mathbb{R}^{I \times J},
$$

where $\boldsymbol{A}_{r} \in \mathbb{R}^{I \times L}$ and $\boldsymbol{B}_{r} \in \mathbb{R}^{J \times L}$ admit rank $L$. In [7], an upper bound on the reconstruction error of such matrices by (2.5) is provided in the general problem. In particular, this error can be as small as desired if $L$ is large enough, which motivates the above assumption.

Reshaping (2.4) into tensor format yields the following:

$$
\mathcal{Z}=\sum_{r=1}^{R}\left(\boldsymbol{A}_{r} \boldsymbol{B}_{r}^{\boldsymbol{\top}}\right) \otimes \boldsymbol{c}_{r} .
$$

The above model can be seen as an LL1-BTD of the tensor $\mathcal{Z}$ with factors $\boldsymbol{A}=\left[\boldsymbol{A}_{1}, \ldots, \boldsymbol{A}_{r}\right]$, $\boldsymbol{B}=\left[\boldsymbol{B}_{1}, \ldots, \boldsymbol{B}_{r}\right]$ and $\boldsymbol{C}=\left[\boldsymbol{c}_{1}, \ldots, \boldsymbol{c}_{r}\right]$ related to the mixing factors.

State-of-the-art unmixing algorithms aim at recovering $\left\{\boldsymbol{S}_{r}=\boldsymbol{A}_{r} \boldsymbol{B}_{r}^{\top}\right\}_{r=1}^{R}$ and $\boldsymbol{C}$ from the mixed pixels in $\mathcal{Z}$. Here, since $\mathcal{Z}$ is unknown and only $\mathcal{Y}_{H}$ is observed with high spectral resolution, these algorithms are only able to recover spatially-degraded versions of the abundance maps [13], namely

$$
\boldsymbol{P}_{1} \boldsymbol{S}_{r} \boldsymbol{P}_{2}^{\top} \in \mathbb{R}^{I_{H} \times J_{H}} \text { for } r \in\{1, \ldots, R\} .
$$

Differently from those works, fusion of an HSI with an MSI with high spatial resolution allows us to seek for abundance maps at a higher spatial resolution.

In Figure 1, our model and the joint unmixing-and-HSR strategy are summarized. Since the true SRIs $\mathcal{Z}$ and $\widetilde{\mathcal{Z}}$ are unknown, we utilize the fusion framework to decompose the HSI and MSI into interpretable mixing factors. While high-resolution spectra can be obtained from the HSI, high-resolution abundance maps can only be obtained from the MSI. The fusion framework allows to exploit fully the information contained in the observations. Once the mixing factors have been retrieved, the estimated SRI $\widehat{\mathcal{Z}}$ can be approximated using the LL1-BTD ${ }^{\ddagger}$. However, the spectral variability has to be modeled first.

2.3. Modeling spectral variability. In traditional unmixing applications, which only deal with a single SRI, spectral variability defines the fact that the spectrum of a material (e.g., grass or soil) changes from pixel to pixel. This sort of spectral variability is widely considered in the literature; see e.g., [44] and references therein. However, two different images are

\footnotetext{
${ }^{\dagger}$ In some traditional unmixing methods (see e.g. [36]), the sum-to-one constrained abundance matrices is also enforced. However, spatial illumination changes frequently introduce scaling variations in each pixel. Moreover, non-negativity constraints can be transformed equivalently to generalized sum-to-one constraint, as specified in [26]. As a result, we do not consider this additional constraint in this work.

${ }^{\ddagger}$ It should be mentioned that this framework is different from a two-step procedure which would i) recover the SRI $\widehat{\mathcal{Z}}$ from data fusion, then ii) run a traditional unmixing algorithm with the estimated SRI.
} 


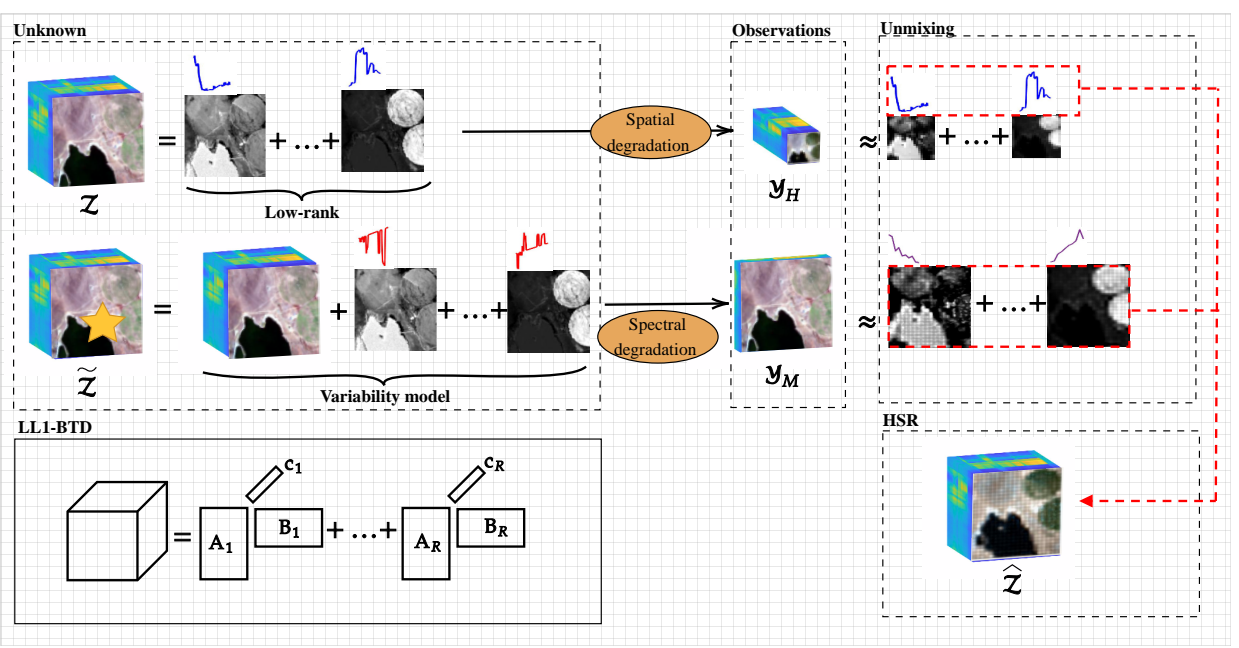

Figure 1. Summary of the model and resolution strategy.

257 where $\widetilde{\boldsymbol{c}}_{r}=\boldsymbol{\psi}_{r}+\boldsymbol{c}_{r}$ is the $r$-th column of $\widetilde{\boldsymbol{C}}$. the MSI as (2.1), we propose to use an equivalent additive model: are able to represent arbitrary endmember variations.

considered in our framework. The variability between their corresponding spectra can be more significant, since their acquisition conditions may be very different. In this paper, we adopt a simple model for spectral variability, which characterizes which wavelengths are more impacted by different acquisition conditions for each material in the images. Although not describing pixel-by-pixel spectral variability, this model is appropriate to describe variations due to different acquisition conditions and will allow us to obtain recovery guarantees.

In [25], the generalized linear mixing model was proposed to model the spectra underlying

$$
\widetilde{\boldsymbol{C}}=\psi_{\text {multi }} \bullet \boldsymbol{C}
$$

where $\boldsymbol{\psi}_{\text {multi }} \in \mathbb{R}^{K \times R}$ is a matrix of positive scaling factors. In this paper, motivated by model

$$
\widetilde{\boldsymbol{C}}=\psi+C,
$$

where $\boldsymbol{\psi} \in \mathbb{R}^{K \times R}$ is different from $\boldsymbol{\psi}_{\text {multi }}$. The choice of the additive variability model (2.9) allows us to keep the spectral variability explicit in $\boldsymbol{\psi}$. Moreover, both models (2.8) and (2.9)

Since we allow only spectral variability to be present, the variability tensor $\boldsymbol{\Psi}$ also admits an LL1-BTD with the same factors $\boldsymbol{A}$ and $\boldsymbol{B}$ as for the SRI $\mathcal{Z}$, but with spectral factor $\boldsymbol{\psi} \in \mathbb{R}^{K \times R}$ representing the spectral variations. This allows to write $\widetilde{\mathcal{Z}}$ as

$$
\widetilde{\mathcal{Z}}=\underbrace{\sum_{r=1}^{R}\left(\boldsymbol{A}_{r} \boldsymbol{B}_{r}^{\boldsymbol{\top}}\right) \otimes \boldsymbol{c}_{r}}_{\mathcal{Z}}+\underbrace{\sum_{r=1}^{R}\left(\boldsymbol{A}_{r} \boldsymbol{B}_{r}^{\boldsymbol{\top}}\right) \otimes \boldsymbol{\psi}_{r}}_{\boldsymbol{\Psi}}=\sum_{r=1}^{R}\left(\boldsymbol{A}_{r} \boldsymbol{B}_{r}^{\boldsymbol{\top}}\right) \otimes \widetilde{\boldsymbol{c}}_{r},
$$


From the above formulations, we can finally express (2.3) as a coupled LL1-BTD:

$$
\left\{\begin{array}{l}
\mathcal{Y}_{H}=\sum_{r=1}^{R}\left(\boldsymbol{P}_{1} \boldsymbol{A}_{r}\left(\boldsymbol{P}_{2} \boldsymbol{B}_{r}\right)^{\mathbf{T}}\right) \otimes \boldsymbol{c}_{r}+\mathcal{E}_{H} \\
\mathcal{Y}_{M}=\sum_{r=1}^{R}\left(\boldsymbol{A}_{r} \boldsymbol{B}_{r}^{\top}\right) \otimes \boldsymbol{P}_{3} \widetilde{\boldsymbol{c}}_{r}+\mathcal{E}_{M}
\end{array}\right.
$$

Thus the joint unmixing-and-HSR problem consists in finding the LL1 factors $\left\{\boldsymbol{A}_{r} \boldsymbol{B}_{r}^{\top}\right\}_{r=1}^{R}$, $\boldsymbol{C}, \widetilde{\boldsymbol{C}}$ under the assumption of (2.11), subject to the constraints

$$
\left\{\boldsymbol{A}_{r} \boldsymbol{B}_{r}^{\top}\right\}_{r=1}^{R} \geq \mathbf{0}, \boldsymbol{C} \geq \mathbf{0}, \widetilde{\boldsymbol{C}} \geq \mathbf{0} .
$$

3. Recoverability analysis. The unmixing-and-HSR problem aims at recovering an SRI $\mathcal{Z}$ and a variability tensor $\boldsymbol{\Psi}$ underlying the HSI in MSI, admitting a coupled LL1-BTD as in (2.11) under the constraints (2.12). The mixing factors underlying the images must also be recovered uniquely. In this section, we provide a noiseless recovery analysis ${ }^{\S}$ for the SRI $\mathcal{Z}$ and variability tensor $\boldsymbol{\Psi}$. We show that our results hold for both image recovery and estimation of the mixing factors. Although the following results are inspired by those of $[54,13]$, there exist two main differences in our work. First, differently from [13], our model accounts for variability. Second, we explicitly address unique recovery of the low-rank LL1 factors as mixing factors, which was not addressed in [13].

Theorem 3.1. Assume that the SRIs $\mathcal{Z}$ and $\widetilde{\mathcal{Z}}$ admit BTDs as in (2.6) and (2.10), respectively, that the HSI and MSI follow the coupled model (2.11), and that $\mathcal{E}_{H}, \mathcal{E}_{M}=\mathbf{0}$. Suppose that $\left\{\boldsymbol{A}_{r}, \boldsymbol{B}_{r}\right\}_{r=1}^{R}, \boldsymbol{C}, \widetilde{\boldsymbol{C}}$ are drawn from any absolutely continuous joint distributions and that $\boldsymbol{P}_{1}, \boldsymbol{P}_{2}, \boldsymbol{P}_{3}$ are full row rank. Let $\left\{\boldsymbol{A}_{r}^{*}, \boldsymbol{B}_{r}^{*}\right\}_{r=1}^{R}, \boldsymbol{C}^{*}, \widetilde{\boldsymbol{C}}^{*}$ denote any solution to the unmixingand-HSR problem. Then with probability one, the true $S R I \mathcal{Z}$ and degraded $S R I \tilde{\mathcal{Z}} \bullet_{3} \boldsymbol{P}_{3}$ are uniquely recovered by

$$
\mathcal{Z}=\sum_{r=1}^{R}\left(\boldsymbol{A}_{r}^{*}\left(\boldsymbol{B}_{r}^{*}\right)^{\boldsymbol{\top}}\right) \otimes \boldsymbol{c}_{r}^{*}, \quad \widetilde{\mathcal{Z}} \bullet_{3} \boldsymbol{P}_{3}=\sum_{r=1}^{R}\left(\boldsymbol{A}_{r}^{*}\left(\boldsymbol{B}_{r}^{*}\right)^{\boldsymbol{\top}}\right) \bullet_{3} \boldsymbol{P}_{3} \widetilde{\boldsymbol{c}}_{r}^{*},
$$

if $I_{H} J_{H} \geq L R, I J \geq L^{2} R$ and

$$
\min \left(\left\lfloor\frac{I}{L}\right\rfloor, R\right)+\min \left(\left\lfloor\frac{J}{L}\right\rfloor, R\right)+\min \left(K_{M}, R\right) \geq 2 R+2 .
$$

Moreover, the abundance maps and spectral signatures represented by the LL1 factors $\left\{\boldsymbol{S}_{r}^{*}=\boldsymbol{A}_{r}^{*}, \boldsymbol{B}_{r}^{*}\right\}_{r=1}^{R}, \boldsymbol{C}^{*}, \boldsymbol{P}_{3} \widetilde{\boldsymbol{C}}^{*}$ are recovered uniquely up to permutation and scaling ambiguities.

Let us first recall the following lemma:

Lemma 3.2. [27, Lemma 1] Let us denote $\widetilde{\boldsymbol{A}}=\boldsymbol{P} \boldsymbol{A} \in \mathbb{R}^{I^{\prime} \times L}$, where $\boldsymbol{P} \in \mathbb{R}^{I^{\prime} \times I}$ is full row rank and $\boldsymbol{A} \in \mathbb{R}^{I \times L}$ is drawn from any absolutely continuous joint distribution. Then $\widetilde{\boldsymbol{A}}$ follows an absolutely continuous joint distribution.

We can now derive the proof for Theorem 3.1.

\footnotetext{
${ }^{\S}$ See also $[45, \mathrm{~S} .1]$ that proposes exact recovery conditions based on the LL1-BTD, from a tensor completion perspective.
} 
Proof. Let $\left\{\boldsymbol{A}_{r}, \boldsymbol{B}_{r}\right\}_{r=1}^{R}, \boldsymbol{C}, \widetilde{\boldsymbol{C}}$ denote the groundtruth factors of the SRI tensors and let $\left\{\boldsymbol{A}_{r}^{*}, \boldsymbol{B}_{r}^{*}\right\}_{r=1}^{R}, \boldsymbol{C}^{*}, \widetilde{\boldsymbol{C}}^{*}$ denote a solution to problem the unmixing-and-HSR problem under constraints (2.12). Moreover, let $\left\{\boldsymbol{S}_{r}\right\}_{r=1}^{R}$ denote the groundtruth abundance maps and $\left\{\boldsymbol{S}_{r}^{*}\right\}_{r=1}^{R}=\left\{\boldsymbol{A}_{r}^{*}\left(\boldsymbol{B}_{r}^{*}\right)^{\top}\right\}_{r=1}^{R}$. Then for $\mathcal{E}_{H}, \mathcal{E}_{M}=\mathbf{0}$, it holds that

$$
\begin{aligned}
& \mathcal{Y}_{H}=\sum_{r=1}^{R}\left(\boldsymbol{P}_{1} \boldsymbol{A}_{r}\left(\boldsymbol{P}_{2} \boldsymbol{B}_{r}\right)^{\boldsymbol{\top}}\right) \otimes \boldsymbol{c}_{r}=\sum_{r=1}^{R}\left(\boldsymbol{P}_{1} \boldsymbol{A}_{r}^{*}\left(\boldsymbol{P}_{2} \boldsymbol{B}_{r}^{*}\right)^{\boldsymbol{\top}}\right) \otimes \boldsymbol{c}_{r}^{*}, \\
& \mathcal{Y}_{M}=\sum_{r=1}^{R} \boldsymbol{S}_{r} \otimes \boldsymbol{P}_{3} \widetilde{\boldsymbol{c}}_{r}=\sum_{r=1}^{R} \boldsymbol{S}_{r}^{*} \otimes \boldsymbol{P}_{3} \widetilde{\boldsymbol{c}}_{r}^{*} .
\end{aligned}
$$

Since by assumption, $\left\{\boldsymbol{A}_{r}, \boldsymbol{B}_{r}\right\}_{r=1}^{R}, \boldsymbol{C}, \widetilde{\boldsymbol{C}}$ are drawn from absolutely continuous joint distributions and $\boldsymbol{P}_{1}, \boldsymbol{P}_{2}$ and $\boldsymbol{P}_{3}$ are full row rank, it follows from Lemma 3.2 that $\left\{\boldsymbol{P}_{1} \boldsymbol{A}_{r}, \boldsymbol{P}_{2} \boldsymbol{B}_{r}\right\}_{r=1}^{R}$, $\boldsymbol{P}_{3} \widetilde{\boldsymbol{C}}$ follow certain absolutely continuous joint distributions.

Therefore, by Theorem 1.5, the LL1-BTD of $\mathcal{Y}_{M}$ is essentially unique almost surely if $I J \geq L^{2} R$ and

$$
\min \left(\left\lfloor\frac{I}{L}\right\rfloor, R\right)+\min \left(\left\lfloor\frac{J}{L}\right\rfloor, R\right)+\min \left(K_{M}, R\right) \geq 2 R+2 .
$$

This means that

$$
\boldsymbol{S}^{*}=\boldsymbol{S} \Pi \boldsymbol{\Lambda}, \quad \boldsymbol{P}_{3} \widetilde{\boldsymbol{C}}^{*}=\boldsymbol{P}_{3} \widetilde{\boldsymbol{C}} \Pi \Lambda^{-1},
$$

where $\boldsymbol{\Pi}$ is a permutation matrix and $\boldsymbol{\Lambda}$ is a non-singular diagonal scaling matrix.

Next, let us define $\widetilde{\boldsymbol{S}}=\left(\boldsymbol{P}_{2} \otimes \boldsymbol{P}_{1}\right) \boldsymbol{S}$. We can see that $\widetilde{\boldsymbol{S}}^{*}=\widetilde{\boldsymbol{S}} \boldsymbol{\Pi} \boldsymbol{\Lambda}$, where $\widetilde{\boldsymbol{S}}^{*}=$ $\left(\boldsymbol{P}_{2} \otimes \boldsymbol{P}_{1}\right) \boldsymbol{S}^{*}$. From [11, Lemma 3.3] and the proof of [13, Theorem II], $\widetilde{\boldsymbol{S}}$ has full column rank almost surely if $I_{H} J_{H} \geq L R$.

Let us continue by considering $\boldsymbol{Y}_{H}^{(3)}$. From (3.1), we have

$$
\boldsymbol{Y}_{H}^{(3)}=\widetilde{\boldsymbol{S}} \boldsymbol{C}^{\top}=\widetilde{\boldsymbol{S}}^{*}\left(\boldsymbol{C}^{*}\right)^{\top}=\widetilde{\boldsymbol{S}} \boldsymbol{\Pi} \boldsymbol{\Lambda}\left(\boldsymbol{C}^{*}\right)^{\top} .
$$

Since $\widetilde{\boldsymbol{S}}$ has full column rank, we thus have

$$
C^{*}=C \Pi \Lambda^{-1} \text {. }
$$

Following (3.3) and (3.4), the LL1 factors $\boldsymbol{S}, \boldsymbol{C}, \boldsymbol{P}_{3} \widetilde{\boldsymbol{C}}$ are recovered uniquely up to permutation and scaling ambiguities by $\boldsymbol{S}^{*}, \boldsymbol{C}^{*}$ and $\boldsymbol{P}_{3} \widetilde{\boldsymbol{C}}^{*}$, respectively.

Finally, we can express the third unfolding of the SRI $\mathcal{Z}$ and degraded $\widetilde{\mathcal{Z}} \bullet_{3} \boldsymbol{P}_{3}$ as

$$
\boldsymbol{Z}^{(3)}=\boldsymbol{S}^{*}\left(\boldsymbol{C}^{*}\right)^{\top},\left(\widetilde{\mathcal{Z}} \bullet_{3} \boldsymbol{P}_{3}\right)^{(3)}=\boldsymbol{S}^{*}\left(\boldsymbol{P}_{3} \widetilde{\boldsymbol{C}}^{*}\right)^{\top},
$$

which are the third unfoldings of the tensors in (3.1)-(3.2).

Remark 3.3. In the proof for Theorem 3.1, we can see that the low-rank factors $\boldsymbol{C}, \boldsymbol{P}_{3} \widetilde{\boldsymbol{C}}$ and $\boldsymbol{S}$ can be uniquely identified up to permutation and scaling ambiguities. This means that they can be interpreted as mixing factors underlying $\mathcal{Z}$ and $\widetilde{\mathcal{Z}} \bullet_{3} \boldsymbol{P}_{3}$. Hence Theorem 3.1 proposes unique recovery conditions for both the fusion and unmixing parts of the problem. 
Theorem 3.1 indicates that we can only recover $\boldsymbol{P}_{3} \widetilde{\boldsymbol{C}}$ uniquely, up to permutation and scaling ambiguities. Following (2.9), the variability matrix $\boldsymbol{\psi}$ can only be recovered from the MSI up to the spectral degradation $\boldsymbol{P}_{3}$ as

$$
\boldsymbol{P}_{3} \psi=\boldsymbol{P}_{3}(\widetilde{C}-\boldsymbol{C}) .
$$

Thus the proposed model only allows to recover uniquely a spectrally-degraded version of the variability tensor, that is, $\boldsymbol{\Psi} \bullet{ }_{3} \boldsymbol{P}_{3}$.

Remark 3.4. In practice, there exist cases where spatial degradation is unknown; previous tensor-based HSR methods [27, 38, 13] proposed unique noiseless recovery conditions for the SRI in this scenario, also referred to as "spatially-blind". However, such methods cannot be envisioned with the proposed approach. Conversely, since knowledge of $\boldsymbol{P}_{3}$ is not required to establish the above theorem, it is possible to seek for "spectrally-blind" algorithms, that do not require the spectral degradation matrix $\boldsymbol{P}_{3}$.

4. Algorithms. In this section, we propose two algorithms based on the LL1-BTD. The first one is unconstrained and solves the HSR problem only. The second one enforced nonnegativity constraints on the factors of the mixing model and proposes a solution to the joint unmixing-and-HSR problem.

4.1. Unconstrained optimization. In the remaining of this paper, for simplicity, we denote $\widetilde{\boldsymbol{C}}_{M}=\left[\widetilde{\boldsymbol{c}}_{M, 1}, \ldots, \widetilde{\boldsymbol{c}}_{M, R}\right]=\boldsymbol{P}_{3} \widetilde{\boldsymbol{C}}$ and $\boldsymbol{\psi}_{M}=\boldsymbol{P}_{3} \boldsymbol{\psi}$. Regarding super-resolution, we only aim at recovering the SRI $\mathcal{Z}$ and variability tensor $\boldsymbol{\Psi}$. In this framework, the latent LL1 factors do not need to be interpretable. Thus, we can consider unconstrained optimization. As in [27], one possible approach for solving the HSR problem is to consider the following optimization problem:

$$
\underset{\boldsymbol{A}, \boldsymbol{B}, \boldsymbol{C}, \widetilde{\boldsymbol{C}}_{M}}{\operatorname{minimize}} \mathcal{J}\left(\boldsymbol{A}, \boldsymbol{B}, \boldsymbol{C}, \widetilde{\boldsymbol{C}}_{M}\right)+\mu\left\|\widetilde{\boldsymbol{C}}_{M}-\left(\boldsymbol{P}_{3} \boldsymbol{C}+\boldsymbol{\psi}_{M}\right)\right\|_{F}^{2},
$$

s. to $\left\|\boldsymbol{c}_{r}\right\|_{2}=1,\left\|\widetilde{\boldsymbol{c}}_{M, r}\right\|_{2}=1$, where

$$
\mathcal{J}\left(\boldsymbol{A}, \boldsymbol{B}, \boldsymbol{C}, \widetilde{\boldsymbol{C}}_{M}\right)=\left\|\mathcal{Y}_{H}-\sum_{r=1}^{R}\left(\boldsymbol{P}_{1} \boldsymbol{A}_{r}\left(\boldsymbol{P}_{2} \boldsymbol{B}_{r}\right)^{\top}\right) \otimes \boldsymbol{c}_{r}\right\|_{F}^{2}+\lambda\left\|\mathcal{Y}_{M}-\sum_{r=1}^{R}\left(\boldsymbol{A}_{r} \boldsymbol{B}_{r}^{\top}\right) \otimes \widetilde{\boldsymbol{c}}_{M, r}\right\|_{F}^{2},
$$

and $\lambda$ is a balance parameter that controls the weights on the HSI and MSI ${ }^{\top}$ The regularization parameter $\mu$ controls the weight on the structural constraint $\widetilde{\boldsymbol{C}}_{M}=\boldsymbol{P}_{3} \boldsymbol{C}+\boldsymbol{\psi}_{M}$. The unit norm constraints on the columns of $\boldsymbol{C}$ and $\widetilde{\boldsymbol{C}}_{M}$ are enforced to avoid convergence issues, and are addressed during optimization with a projected gradient approach. Since (4.1) is a non-convex cost function, we adopt a block coordinate descent scheme: the latent factors are updated sequentially by solving unconstrained convex quadratic programs.

Below, we provide the framework of the unconstrained algorithm, named BTD-Var. The updates for $\boldsymbol{A}, \boldsymbol{B}$ and $\boldsymbol{C}$ can be seen as generalized Sylvester equations and solved by efficient solvers, for instance, Hessenberg-Schur or Bartels-Stewart algorithms; see [43] for a full overview. The update for $\widetilde{\boldsymbol{C}}_{M}$ is solved using normal equations. Please refer to Appendix A for a full derivation.

\footnotetext{
『 As in previous works [27]-[13], we consider that $\lambda=1$ in our experiments.
} 


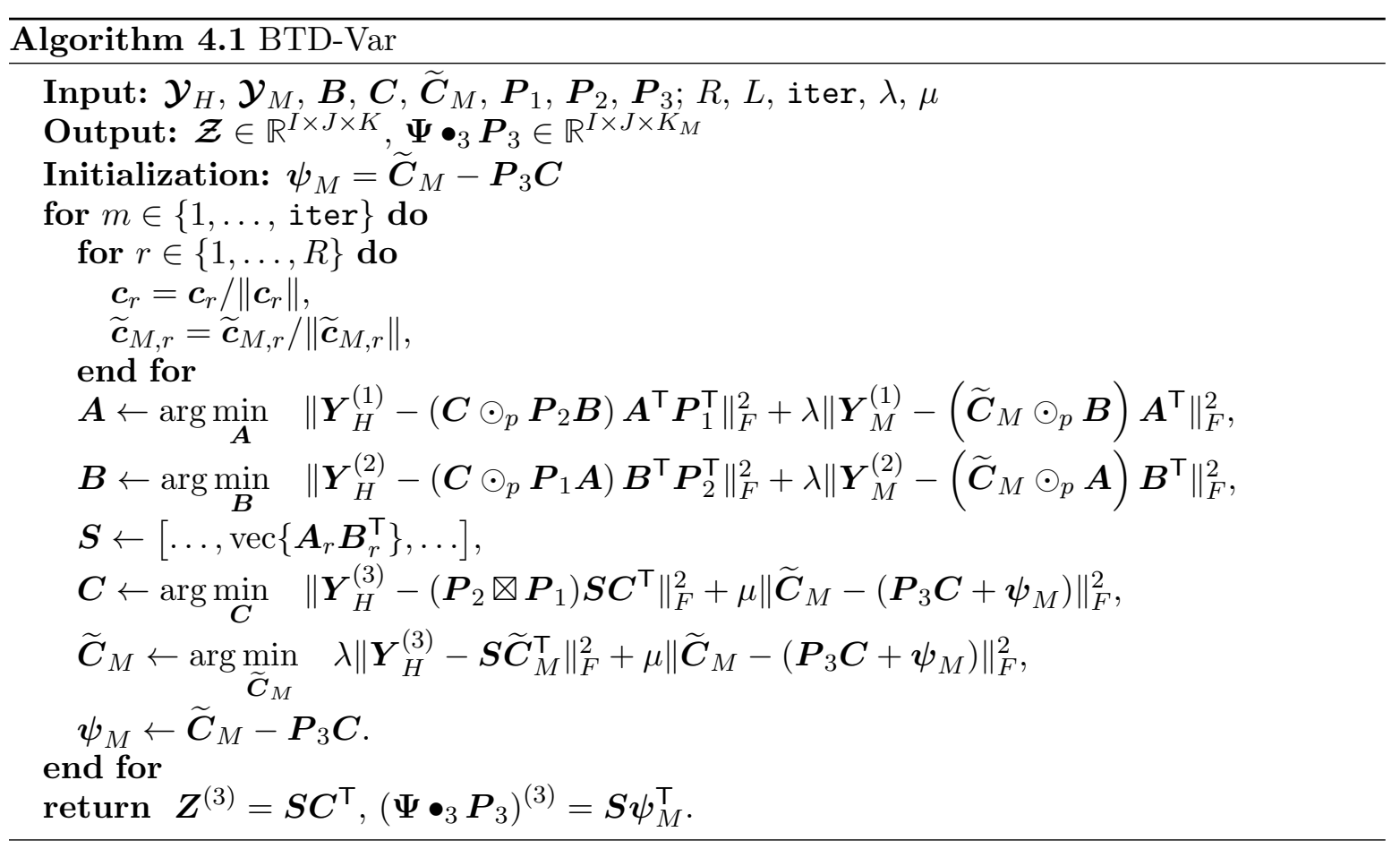

The computational cost per iteration of BTD-Var can be decomposed as follows:

- $\mathcal{O}\left(I^{3}+J^{3}+K^{3}+L^{3} R^{3}\right)$ for solving the Sylvester equations;

- $\mathcal{O}\left(I J K_{M} R+I_{H} J_{H} K R\right)$ for computing the right-hand side in the least squares subproblem.

4.2. Constrained optimization. Although BTD-Var allows for reconstruction of $\mathcal{Z}$ and $\boldsymbol{\Psi}$, it is not guaranteed that its result can be interpretable in a mixing model. Indeed, nonnegativity constraints must be imposed on factors $\boldsymbol{C}$ and $\widetilde{\boldsymbol{C}}_{M}$ to provide them with physical meaning. Differently from [53], we also impose non-negativity on $\left\{\boldsymbol{S}_{r}\right\}_{r=1}^{R}$, rather than on the individual factors $\boldsymbol{A}_{r}$ and $\boldsymbol{B}_{r}$. This way, $\boldsymbol{c}_{r}$ and $\widetilde{\boldsymbol{c}}_{M, r}$ (resp. $\boldsymbol{S}_{r}$ ) can be seen as spectral signatures (resp. abundance maps) of the underlying SRI $\mathcal{Z}$ and MSI $\mathcal{Y}_{M}$.

The resulting constrained optimization problem is:

$$
\begin{aligned}
& \underset{\boldsymbol{A}, \boldsymbol{B},\left\{\boldsymbol{S}_{r}\right\}_{r=1}^{R}, \boldsymbol{C}, \widetilde{\boldsymbol{C}}_{M}}{\operatorname{minimize}} \mathcal{J}\left\|\mu \widetilde{\boldsymbol{C}}_{M}-\left(\boldsymbol{P}_{3} \boldsymbol{C}+\boldsymbol{\psi}_{M}\right)\right\|_{F}^{2}+\gamma\left\|\boldsymbol{S}_{r}-\boldsymbol{A}_{r} \boldsymbol{B}_{r}^{\top}\right\|_{F}^{2} \\
& \text { s. to }\left\{\boldsymbol{S}_{r}=\boldsymbol{A}_{r} \boldsymbol{B}_{r}^{\top}\right\}_{r=1}^{R} \geq \mathbf{0}, \boldsymbol{C} \geq \mathbf{0}, \widetilde{\boldsymbol{C}}_{M} \geq \mathbf{0},\left\|\boldsymbol{c}_{r}\right\|_{2}=1,\left\|\widetilde{\boldsymbol{c}}_{M, r}\right\|_{2}=1,
\end{aligned}
$$

where $\gamma$ is a regularization parameter that controls the weight on the low-rank constraint.

Differently from (4.1), in (4.2)-(4.3) the $\boldsymbol{S}_{r}$ factors are no longer latent variables, and are subject to non-negativity constraints. Such constraints can be handled by using alternating direction method of multipliers [9, 23]. As in [23], a non-negativity constraint is relaxed by considering the surrogate $\iota_{+}(\cdot)$ (see Appendix B). Algorithm 4.2 presents the optimization framework for (4.2)-(4.3).

The computational cost per-iteration of CNN-BTD-Var is:

- $\mathcal{O}\left(I^{3}+J^{3}+K^{3}+L^{3} R^{3}\right)$ for solving $\boldsymbol{A}, \boldsymbol{B}$ and $\boldsymbol{C}$; 


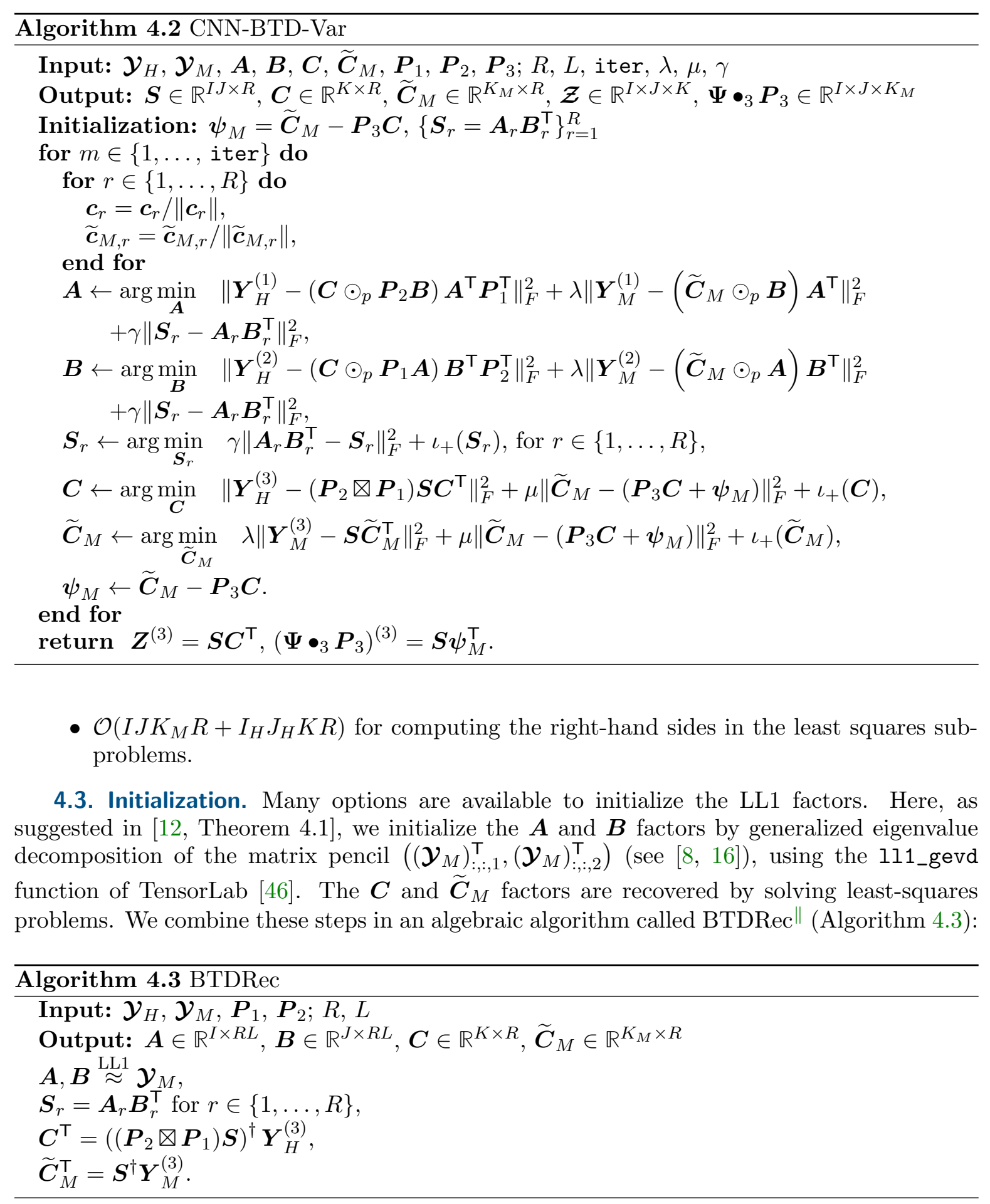

\footnotetext{
"echoing the initialization algorithm in [27] (called TenRec)
} 
5. Experiments for image recovery. All simulations were run on a MacBook Pro with $2.3 \mathrm{GHz}$ Intel Core i5 and 16GB RAM. For basic tensor operations we used TensorLab 3.0 [46]. The code was implemented in MATLAB and is available online at https://github.com/ cprevost4/LL1_HSR_HU.

5.1. Degradation model. The real SRI and MSI were acquired with the same spatial resolutions by the AVIRIS and Sentinel-2A instruments at different time instants, resulting in variability between the images. The spectral bands of $\mathcal{Z}$ and $\mathcal{Y}_{M}$ were normalized such that the 0.999 intensity quantile corresponded to a value of 1 . This ensured that the unit norm constraint for the columns of $\boldsymbol{C}$ and $\widetilde{\boldsymbol{C}}_{M}$ did not cause any convergence problems. The HSI was obtained by spatial degradation of $\mathcal{Z}$ by $\boldsymbol{P}_{1}$ and $\boldsymbol{P}_{2}$, i.e., the SRI $\mathcal{Z}$ and the MSI $\mathcal{Y}_{M}$ represented images of the same scene acquired on board of different missions, and $\widetilde{\mathcal{Z}}$ was unknown. Afterwards, the SRI $\mathcal{Z}$ was denoised (as described in [40]) to yield the high-SNR reference image [50]. We also conducted experiments in a "no-variability" scenario, i.e. we considered that the HSI and MSI were obtained by spatial (resp. spectral) degradation of the same SRI $\mathcal{Z}$.

For spatial degradation, we followed the commonly used Wald's protocol [47]. The matrices $\boldsymbol{P}_{1}, \boldsymbol{P}_{2}$ were computed with a separable Gaussian blurring kernel of size $q=9$. Downsampling was performed along each spatial dimension with a ratio $d=4$ between the SRI and HSI, as in previous works [27]-[13]. Refer to Appendix C for more details on the construction of $\boldsymbol{P}_{1}$, $\boldsymbol{P}_{2}$. White Gaussian noise with $30 \mathrm{~dB}$ SNR was added to the HSI and MSI.

For the spectral degradation matrix $\boldsymbol{P}_{3}$, we used the spectral response functions of two multispectral instruments**. For images with spectral variability, the Sentinel-2 sensors span the electromagnetic spectrum from $412 \mathrm{~nm}$ to $2022 \mathrm{~nm}$ and produced a 10-band MSI corresponding to the wavelengths $433-453 \mathrm{~nm}$ (atmospheric correction), 458-522nm (soil, vegetation), 543-577nm (green peak), 650-680nm (maximum chlorophyll absorption), 698-712nm (red edge), 733-747nm (red edge), 773-793nm (leaf area index, edge of NIR), 785-900nm (leaf area index), $855-875 \mathrm{~nm}$ (NIR plateau), 935-955nm (water vapour absorption). The LANDSAT sensor spanned the spectrum from $400 \mathrm{~nm}$ to $2500 \mathrm{~nm}$ for the HSI and produced a 6 -band MSI corresponding to wavelengths 450-520nm (black), 520-600nm (green), 630-690nm (red), 760-900nm (near-IR), 1550-1750nm (shortwave-IR) and 2050-2350nm (shortwave-IR2). This spectral response was used for real images without spectral variability. The spectral degradation matrix $\boldsymbol{P}_{3}$ was a selection-weighting matrix that selected the common spectral bands of the SRI $\widetilde{\mathcal{Z}}$ and the MSI.

5.2. Metrics. We compared the groundtruth SRI $\mathcal{Z}$ with the recovered SRI $\widehat{\mathcal{Z}}$ obtained by the algorithms. The main performance metric used in comparisons was the reconstruction Signal-to-Noise ratio (R-SNR):

$$
\mathrm{R}-\mathrm{SNR}=10 \log _{10}\left(\frac{\|\mathcal{Z}\|_{F}^{2}}{\|\widehat{\mathcal{Z}}-\mathcal{Z}\|_{F}^{2}}\right)
$$

\footnotetext{
${ }^{* *}$ available for download at https://earth.esa.int/web/sentinel/user-guides/sentinel-2-msi/ document-library/-/assetpublisher/Wk0TKajiISaR/content/sentinel-2a-spectral-responses and https: //landsat.gsfc.nasa.gov/landsat-8/.
} 
In addition to R-SNR, we considered different metrics described below:

$$
\mathrm{CC}=\frac{1}{I J K}\left(\sum_{k=1}^{K} \rho\left(\mathcal{Z}_{:,:, k}, \widehat{\mathcal{Z}}_{:,:, k}\right)\right),
$$

where $\rho(\cdot, \cdot)$ is the Pearson correlation coefficient between the estimated and original spectral slices;

$$
\text { ERGAS }=\frac{100}{d} \sqrt{\frac{1}{I J K} \sum_{k=1}^{K} \frac{\left\|\widehat{\mathcal{Z}}_{:,, k}-\mathcal{Z}_{:,:, k}\right\|_{F}^{2}}{\mu_{k}^{2}}},
$$

where $\mu_{k}^{2}$ is the mean value of $\widehat{\mathcal{Z}}_{:,,, k}$. ERGAS represents the relative dimensionless global error between the SRI and the estimate, which is the root mean-square error averaged by the size of the SRI. We also used Spectral Angle Distance (SAD):

$$
\mathrm{SAD}=\frac{1}{R} \sum_{r=1}^{R} \arccos \left(\frac{\boldsymbol{c}_{r}^{\top} \widehat{\boldsymbol{c}}_{r}}{\left\|\boldsymbol{c}_{r}\right\|_{2}\left\|\widehat{\boldsymbol{c}}_{r}\right\|_{2}}\right),
$$

which computes the spectral angle distance between original and estimated spectra, and can be used to assess unmixing performance as well. Performance for recovery of the abundance maps was assessed using the root mean-squared error between reference $\boldsymbol{S}$ and estimate $\widehat{\boldsymbol{S}}$ :

$$
\operatorname{RMSE}=\frac{1}{R} \sum_{r=1}^{R} \sqrt{\left.\frac{1}{I J} \sum_{d=1}^{I J}\left(\left(\boldsymbol{S}_{r}\right)_{d}-\left(\widehat{\boldsymbol{S}}_{r}\right)_{d}\right)\right)^{2}} .
$$

Finally, we considered the computational time for each algorithm, given by the tic and toc functions of MATLAB.

5.3. Recovery of the SRI and variability tensor. In this subsection, we assessed the performances of Algorithm 4.1 (BTD-Var) and Algorithm 4.2 (CNN-BTD-Var) for reconstruction of the SRI $\mathcal{Z}$ and degraded variability tensor $\boldsymbol{\Psi} \bullet_{3} \boldsymbol{P}_{3}$. We ran our algorithms with 20 outer iterations at most and 5 inner iterations for CNN-BTD-Var. For initialization, out of 20 trials of BTDRec we picked the one that provided the best reconstruction of the HSI and MSI. For CNN-BTD-Var, we used $\mu=\gamma=1$. We chose the hyperparameters $R$ and $L$ jointly. While $L$ was as large as possible inside the identifiability region provided by Theorem 3.1, $R$ was selected according to the real number of endmembers, when possible. Other rank-selecting algorithms are available for hyperspectral images, see e.g., [2, 19]. Due to page limitations, please refer to the Supplementary materials for a thorough discussion on the choice of $R$ and $L$.

For the SRI $\mathcal{Z}$, we compared our results to matrix-based approaches, including HySure [42], CNMF [51] and GLP-HS [1]. We also considered tensor methods, namely STEREO [27] for CP decomposition, SCOTT [38] for Tucker and CNN-BTD [53], which is a coupled LL1-based algorithm that does not account for spectral variability. Finally, we considered matrix and tensor methods accounting for variability, namely FuVar [4] (a matrix-based algorithm based on the generalized linear mixing model), CT-STAR and CB-STAR [6], which are 
tensor approaches based on multilinear decomposition accounting for spectrally and spatially localized changes. Except for CNMF, the baseline algorithms are unable to perform the unmixing task. For Hysure, CNMF, GLP-HS and FuVar, we chose the ranks and regularization parameters according to the original works $[42,51,4]$.

For reconstruction of $\boldsymbol{\Psi} \bullet_{3} \boldsymbol{P}_{3}$, we compared the results of our algorithms with those of CT-STAR and CB-STAR.

5.3.1. Lake Tahoe. The first dataset was Lake Tahoe with $\mathcal{Z} \in \mathbb{R}^{100 \times 80 \times 173}$. The SRI $\mathcal{Z}$ and MSI $\mathcal{Y}_{M}$ were respectively acquired on 2014-10-04 and 2017-10-24 by the Sentinel$2 \mathrm{~A}$ sensor, resulting in high variability in the crops and lake areas). We ran STEREO with $F=30$ and 10 iterations, and SCOTT with $R=(40,40,7)$ as in [6]. We ran CT-STAR with ranks $(18,15,10),(3,3,1)$, and CB-STAR with ranks $(20,20,9),(20,20,4)$. For our algorithms, as well as for CNN-BTD, we chose $R=3, L=20$ and $\lambda=1$. Tables 1 and 2 display the reconstruction metrics and computation time for $\mathcal{Z}$ and $\Psi \bullet_{3} \boldsymbol{P}_{3}$ and all considered algorithms. The two best results of each column are shown in bold.

Table 1

Reconstruction metrics for $\mathcal{Z}$, Lake Tahoe dataset

\begin{tabular}{c|c|c|c|c|c}
\hline Algorithm & R-SNR & CC & SAD & ERGAS & Time \\
\hline BTD-Var & 15.0746 & 0.9384 & 9.7688 & 5.2081 & 2.6390 \\
CNN-BTD-Var & $\mathbf{1 6 . 1 3 7 1}$ & $\mathbf{0 . 9 5 1 4}$ & 7.2173 & $\mathbf{4 . 5 9 0 2}$ & 1.2251 \\
STEREO & 5.8368 & 0.75957 & 30.7346 & 15.2801 & 1.2148 \\
SCOTT & 1.918 & 0.50379 & 47.1781 & 23.3815 & $\mathbf{0 . 1 4 7 0 1}$ \\
CNN-BTD & 6.0332 & 0.80003 & 27.7993 & 14.9491 & 1.2826 \\
CNMF & 12.1314 & 0.87494 & 9.2422 & 7.2804 & 1.7442 \\
GLP-HS & 11.7862 & 0.87408 & 11.6106 & 7.6011 & 4.507 \\
HySure & 9.2687 & 0.81256 & 12.8228 & 10.1511 & 7.2761 \\
FuVar & 14.54 & 0.92498 & $\mathbf{6 . 7 0 1 3}$ & 5.528 & 761.3932 \\
CT-STAR & 11.7676 & 0.87843 & 13.3433 & 7.6236 & $\mathbf{0 . 2 0 8 4 9}$ \\
CB-STAR & $\mathbf{1 9 . 2 4 1 3}$ & $\mathbf{0 . 9 7 5 3 9}$ & $\mathbf{6 . 4 6 4 9}$ & $\mathbf{3 . 2 2 3 1}$ & 8.3597
\end{tabular}

Table 2

Reconstruction metrics for $\boldsymbol{\Psi} \bullet_{3} \boldsymbol{P}_{3}$, Lake Tahoe dataset

\begin{tabular}{c|c|c|c|c}
\hline Algorithm & R-SNR & CC & SAD & ERGAS \\
\hline BTD-Var & 13.8652 & 0.8584 & 14.7252 & 11.9947 \\
CNN-BTD-Var & $\mathbf{1 4 . 7 3 4 7}$ & $\mathbf{0 . 8 6 5 4}$ & $\mathbf{9 . 7 9 1 6}$ & $\mathbf{1 1 . 8 2 7 8}$ \\
CT-STAR & 11.4131 & 0.84542 & 17.7857 & 12.8223 \\
CB-STAR & $\mathbf{1 6 . 6 5 9 9}$ & $\mathbf{0 . 9 4 1 6 1}$ & $\mathbf{1 0 . 4 4 4 2}$ & $\mathbf{7 . 8 5 6 9}$
\end{tabular}

We can see that algorithms accounting for variability provided the best reconstruction metrics: in particular, the high performance of CB-STAR resulted from the fact that the algorithm accounts for spectrally and spatially localized changes. BTD-Var and CNN-BTDVar provided slightly higher metrics than FuVar, but with lower computation time. Among the matrix-based approaches, CNMF showed the best reconstruction performance. Finally, other tensor-based approaches, although fast, yielded worse reconstruction metrics, due to the fact that they did not consider the variability. CB-STAR also provided the best metrics for reconstruction of $\Psi \bullet_{3} \boldsymbol{P}_{3}$. However, its computation time was large. The proposed algorithms showed competitive metrics and even slightly outperformed baseline methods in terms of CC, 
but with slightly higher computation time.

In addition, we plot in Figure 2 the 40th spectral band of the reference and estimated SRI. The proposed approaches recovered the SRI spectral band accurately.
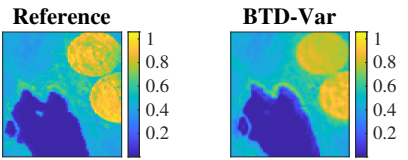

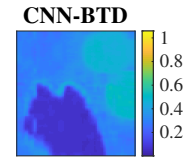

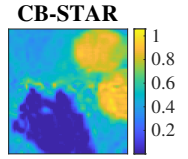

Figure 2. Single spectral band of the SRI, Lake Tahoe dataset

5.3.2. Ivanpah Playa. We also considered Ivanpah Playa with $\mathcal{Z} \in \mathbb{R}^{80 \times 128 \times 173}$ and large acquisition time difference: the SRI and MSI were acquired on 2015-10-26 and 2017-12-17 respectively, by the Sentinel-2 sensor. We ran STEREO with $F=10$ and 10 iterations and SCOTT with $R=(30,30,10)$. We ran CT-STAR with ranks $(10,15,8),(3,3,2)$, and CBSTAR with ranks $(40,40,4),(40,40,5)$. For our algorithms and CNN-BTD, we chose $R=4$ and $L=18$.

Tables 3 and 4 show the reconstruction metrics and computation time.

Table 3

Reconstruction metrics for $\mathcal{Z}$, Ivanpah Playa dataset

\begin{tabular}{c|c|c|c|c|c}
\hline Algorithm & R-SNR & CC & SAD & ERGAS & Time \\
\hline BTD-Var & 19.5964 & 0.8684 & 2.4533 & 2.6223 & 3.2545 \\
CNN-BTD-Var & $\mathbf{2 4 . 7 9 9 1}$ & $\mathbf{0 . 9 5 3 3}$ & 1.5943 & $\mathbf{1 . 4 7 2 5}$ & 2.2159 \\
STEREO & 6.0987 & 0.76283 & 29.0278 & 12.6747 & 0.93975 \\
SCOTT & 2.4445 & 0.34257 & 47.9598 & 19.372 & $\mathbf{0 . 2 6 4 5}$ \\
CNN-BTD & 5.7515 & 0.33492 & 28.7006 & 13.1899 & 11.8775 \\
CNMF & 21.6059 & 0.90114 & $\mathbf{1 . 3 0 1 9}$ & 2.1138 & 2.6656 \\
GLP-HS & 19.433 & 0.86261 & 3.3413 & 2.697 & 5.9218 \\
HySure & 18.4551 & 0.85218 & 3.3249 & 3.0653 & 10.4606 \\
FuVar & 22.0332 & 0.90354 & 1.5062 & 2.0189 & 526.1659 \\
CT-STAR & 21.1186 & 0.88849 & 1.9424 & 2.2386 & $\mathbf{0 . 1 5 3 7 3}$ \\
CB-STAR & $\mathbf{2 5 . 7 1 7 4}$ & $\mathbf{0 . 9 6 0 0 3}$ & $\mathbf{1 . 3 2 6 9}$ & $\mathbf{1 . 3 2 2 8}$ & 8.2923
\end{tabular}

Table 4

Reconstruction metrics for $\mathbf{\Psi} \bullet_{3} \boldsymbol{P}_{3}$, Ivanpah Playa dataset

\begin{tabular}{c|c|c|c|c}
\hline Algorithm & R-SNR & CC & SAD & ERGAS \\
\hline BTD-Var & 19.3624 & 0.7057 & 2.7901 & 39.5265 \\
CNN-BTD-Var & $\mathbf{2 3 . 6 5 5 8}$ & $\mathbf{0 . 9 2 0 7}$ & $\mathbf{1 . 3 8 2 6}$ & $\mathbf{1 5 . 0 0 5 4}$ \\
CT-STAR & 19.3597 & 0.73396 & 2.1977 & 33.853 \\
CB-STAR & $\mathbf{2 3 . 4 8 8 8}$ & $\mathbf{0 . 9 0 8 3 2}$ & $\mathbf{1 . 1 5 6 7}$ & $\mathbf{1 6 . 9 8 1 5}$
\end{tabular}

The best metrics were provided by CB-STAR, then CNN-BTD-Var. BTD-Var had a performance comparable to that of GLP-HS for reconstruction of $\mathcal{Z}$. Its performance was comparable to that of CT-STAR for $\boldsymbol{\Psi} \bullet_{3} \boldsymbol{P}_{3}$. For this dataset as well, the proposed algorithms were faster than some other algorithms, including CNMF, CB-STAR, and FuVar. Other matrix-based approaches also provided satisfying reconstruction. However, STEREO, SCOTT and CNN-BTD provided the worst reconstruction metrics. 
In Figure 3 we plot the 40th spectral band of the reference SRI and the estimated SRI for our algorithms, CNN-BTD, CNMF and CB-STAR for comparison. For this dataset, we can
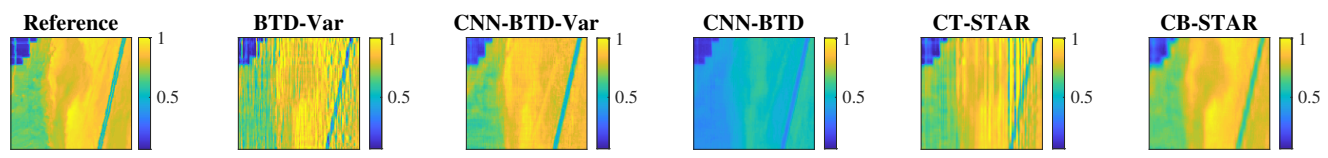

Figure 3. Single spectral band of the SRI, Ivanpah Playa dataset

see that CNN-BTD-Var yielded a better SRI spectral band reconstruction than BTD-Var.

5.3.3. Lockwood. The third dataset we considered was Lockwood with $\mathcal{Z} \in \mathbb{R}^{80 \times 100 \times 173}$. The SRI $\mathcal{Z}$ and MSI were acquired on 2018-08-20 and on 2018-10-19. This was an example where only acquisition variations happened (which affected the image mostly uniformly), thus it illustrated the adequacy of the proposed variability model compared to more general ones proposed in the literature. We ran STEREO with $F=100$ and 10 iterations and SCOTT with $\boldsymbol{R}=(60,60,5)$. We ran CT-STAR with ranks $(30,30,8),(3,3,2)$, and CB-STAR with ranks $(70,70,5),(40,40,3)$. For our algorithms, as well as for CNN-BTD, we chose $R=9$ and $L=16$. The reconstruction metrics are displayed in Tables 5 and 6 .

Table 5

Reconstruction metrics for $\mathcal{Z}$, Lockwood dataset

\begin{tabular}{c|c|c|c|c|c}
\hline Algorithm & R-SNR & CC & SAM & ERGAS & Time (sec) \\
\hline BTD-Var & $\mathbf{2 0 . 1 2 7 3}$ & $\mathbf{0 . 9 1 8 4 3 2}$ & $\mathbf{2 . 9 2 9 2 1}$ & $\mathbf{6 . 3 5 5 6 6}$ & 5.46272 \\
CNN-BTD-Var & $\mathbf{1 9 . 4 8 8 2}$ & $\mathbf{0 . 9 0 6 5 2 5}$ & 3.0299 & $\mathbf{6 . 2 9 1 0 1}$ & 4.11573 \\
STEREO & 6.552 & 0.80196 & 27.3623 & 25.1749 & $\mathbf{1 . 8 8 3 5}$ \\
SCOTT & 2.2276 & 0.79276 & 28.5771 & 45.9608 & $\mathbf{0 . 2 2 2 8}$ \\
CNN-BTD & 6.4909 & 0.81475 & 27.4245 & 25.436 & 2.3082 \\
CNMF & 18.7829 & 0.89063 & $\mathbf{2 . 9 7 6 8}$ & 6.7014 & 4.353 \\
GLP-HS & 18.6734 & 0.88849 & 3.2079 & 6.9979 & 6.8463 \\
HySure & 14.125 & 0.8633 & 4.4044 & 11.6 & 6.9823 \\
CT-STAR & 18.4987 & 0.88287 & 4.571 & 8.2657 & 3.3013 \\
CB-STAR & 19.0751 & 0.89445 & 3.3707 & 7.2926 & 68.0282
\end{tabular}

Table 6

Reconstruction metrics for $\boldsymbol{\Psi} \bullet_{3} \boldsymbol{P}_{3}$, Lockwood dataset.

\begin{tabular}{c|c|c|c|c}
\hline Algorithm & R-SNR & CC & SAM & ERGAS \\
\hline BTD-Var & $\mathbf{1 8 . 8 7 6 8}$ & $\mathbf{0 . 8 1 0 1 7 1}$ & $\mathbf{2 . 5 9 8 6 2}$ & $\mathbf{1 1 . 9 2 5 3}$ \\
CNN-BTD-Var & $\mathbf{1 8 . 3 5 2 3}$ & $\mathbf{0 . 8 1 8 4 2 4}$ & $\mathbf{2 . 7 6 5 3 8}$ & $\mathbf{1 1 . 2 0 9 5}$ \\
CT-STAR & 17.2744 & 0.73293 & 4.1677 & 15.8113 \\
CB-STAR & 17.5513 & 0.7402 & 3.2858 & 13.3116
\end{tabular}

For both $\mathcal{Z}$ and $\boldsymbol{\Psi} \bullet \bullet_{3} \boldsymbol{P}_{3}$, the best reconstruction metrics were generally provided by BTDVar and CNN-BTD-Var. They were followed by CT-STAR and CB-STAR. The slightly better results obtained by our algorithms illustrate the fact that the variability model considered in [6] can represent spatially localized changes, but is not very appropriate or interpretable for 
520 acquisition or illumination variations. In Figure 4 we plot the 40th spectral band of the reference and estimated SRI.
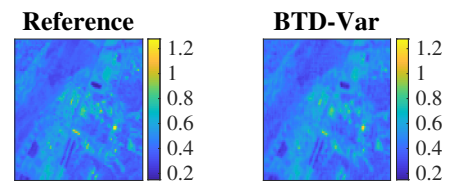
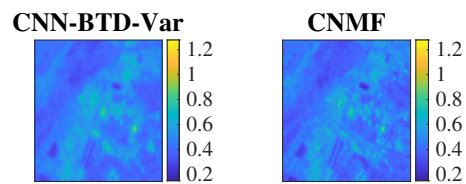

CB-STAR

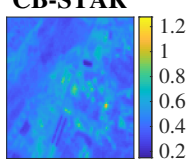

Figure 4. Spectral band no.40 of the SRI, Lockwood.

Table 7

5.4. Recovery without variability. In this subsection, we assessed recovery performance for the SRI $\mathcal{Z}$. We considered that the HSI and MSI are both degraded versions of the same SRI $\mathcal{Z}$. In other words, we suppose that $\mathcal{Z}=\widetilde{\mathcal{Z}}$, hence there is no spectral variability. The dataset we considered was Indian Pines, where $\mathcal{Z} \in \mathbb{R}^{144 \times 144 \times 200}$ was degraded by a LANDSAT sensor for the MSI and a downsampling ratio $d=4$ for the HSI. We ran STEREO with $F=50$, SCOTT with $R=(40,40,6)$ and LL1-based algorithms with $R=6$ and $L=13$. We tuned the other algorithms according to original works. The reconstruction metrics for the SRI are presented in Table 7.

Reconstruction metrics for $\mathcal{Z}$, Indian Pines dataset

\begin{tabular}{c|c|c|c|c|c}
\hline Algorithm & R-SNR & CC & SAD & ERGAS & Time \\
\hline BTD-Var & 26.4299 & 0.8398 & 2.2384 & 1.1750 & 5.8435 \\
CNN-BTD-Var & 25.1166 & 0.8326 & 2.4127 & 1.2675 & 4.2968 \\
STEREO & $\mathbf{2 7 . 6 9}$ & 0.86669 & $\mathbf{1 . 9 4 6 1}$ & $\mathbf{0 . 9 9 9 5 9}$ & 1.8564 \\
SCOTT & 26.2451 & $\mathbf{0 . 8 6 1 9 6}$ & 2.2694 & 1.1208 & $\mathbf{0 . 2 1 0 8 7}$ \\
CNN-BTD & 25.2263 & 0.80949 & 2.5035 & 1.3497 & 24.5326 \\
CNMF & $\mathbf{2 7 . 2 5 5 2}$ & 0.83978 & $\mathbf{1 . 9 5 0 2}$ & 1.2056 & 8.2147 \\
GLP-HS & 26.2837 & 0.83813 & 2.2794 & 1.2918 & 14.2957 \\
HySure & 20.4281 & 0.66661 & 4.4916 & 2.5723 & 25.2202 \\
CT-STAR & 24.0398 & 0.84385 & 2.4839 & 1.3151 & $\mathbf{0 . 1 6 5 2 8}$ \\
CB-STAR & 26.5216 & $\mathbf{0 . 8 6 7 4 9}$ & 2.1265 & $\mathbf{1 . 0 5 5 6}$ & 3.6761
\end{tabular}

The best reconstruction metrics were generally provided by STEREO. The proposed algorithms had performance comparable to that of SCOTT, and computation time comparable to that of CB-STAR. The slightly lower performance of constrained algorithms accounting for variability can be explained by the use of more flexible models. In this specific scenario, other methods based on a more restrictive model fitted the data more tightly. Nonetheless, algorithms accounting for variability offered competitive performance in the "no-variability" case. However, their computation time was usually higher than that of state-of-the-art tensor approaches. In Figure 5 we plot the 40th spectral band of the reference and estimated SRI.

\section{Performance for unmixing of an unknown SRI.}

6.1. Experiments setup. In this section, we assessed the performance of CNN-BTD-Var for unmixing of an unknown SRI on synthetic datasets, and real examples from Section 5.

We compared our results with those of CNMF [51] initialized by VCA [35]. We also considered traditional unmixing algorithms: accelerated multiplicative algorithm (MU-Acc) 

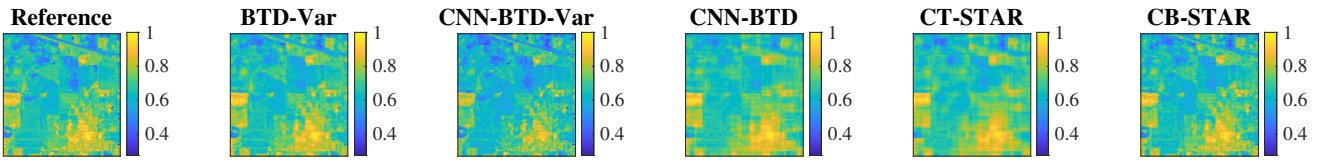

Figure 5. Single spectral band of the SRI, Indian Pines dataset

[21] and BMDR-ADMM [36] (enforcing minimum dispersion constraint on the spectra, and sum-to-one on the abundance maps). We ran these algorithms on the recovered SRI $\widehat{\mathcal{Z}}$ obtained from CB-STAR, which gave the best reconstruction metrics in the previous section. For these algorithms, we chose the parameters according to the original works. We set the number of materials to $R$. Since other fusion algorithms were not designed for unmixing, they were not directly included in this comparison.

For each dataset, we compared the abundance maps and spectral signatures obtained by the algorithms to references obtained by VCA or groundtruth materials. We also assessed the unmixing performance numerically by comparing the SAD, RMSE and computation time for the considered algorithms. The best results of each row are shown in bold in the tables.

6.2. Unmixing with exact LL1 model. We first assessed the unmixing performance with synthetic datasets. We tested our approach in the case where the SRI and variability tensor admit an exact LL1-BTD. Although these datasets did resemble real spectral images, they allowed us to assess unmixing performance in a case where the uniqueness conditions for the non-negative matrix factorization model (see [14, 32]) were not fulfilled.

6.2.1. Generating synthetic datasets. We considered $R=3$ spectral signatures $\boldsymbol{c}_{r}(r \in$ $\{1, \ldots, R\})$ obtained from the Jasper Ridge reference data ${ }^{\dagger \dagger}$, corresponding to vegetation, soil and road materials. The SRI $\mathcal{Z} \in \mathbb{R}^{I \times J \times K}(I=J=90, K=173)$ was split into $L R$ equal blocks in the spatial dimensions, with $L=3$.

Each abundance map $\boldsymbol{S}_{r}(r \in\{1, \ldots, R\})$ was a block matrix with $\frac{I}{L} \times \frac{J}{L}$ blocks. We generated a multiplicative variability matrix $\boldsymbol{\psi}_{\text {multi }}$ with random real entries drawn from the standard uniform distribution in the open interval $[0.9,1.1]$. We then computed the altered spectra $\widetilde{\boldsymbol{C}}=\boldsymbol{\psi}_{\text {multi }} \triangleright \boldsymbol{C}$. The variability matrix $\boldsymbol{\psi}$ that we aimed at recovering was obtained as $\boldsymbol{\psi}=\widetilde{\boldsymbol{C}}-\boldsymbol{C}$ so that it had zero mean.

Formally, we computed the high-resolution tensors as

$$
\mathcal{Z}=\sum_{r=1}^{R} \boldsymbol{S}_{r} \otimes \boldsymbol{c}_{r}, \quad \boldsymbol{\Psi}=\sum_{r=1}^{R} \boldsymbol{S}_{r} \otimes \boldsymbol{\psi}_{r}, \quad \widetilde{\mathcal{Z}}=\mathcal{Z}+\boldsymbol{\Psi} .
$$

The HSI and MSI were obtained by degradation of the SRIs according to model (2.3). For $\boldsymbol{P}_{1}=\boldsymbol{P}_{2}$, we had $q=9$ and $d=3$ so that $I_{H}=J_{H}=30$. For $\boldsymbol{P}_{3}$, we chose the spectral response of the Sentinel-2 MS sensor, which led to $K_{M}=10$.

6.2.2. Separable example. In the first example, we generated a dataset for which the pure pixel assumption was valid. Thus in each $\frac{I}{L} \times \frac{J}{L}$ block, at most one material was active, as indicated by the numerals in the parcel map shown in Table 8. Each block in the parcel map was a patch composed of entries equal to one. The abundance maps resembled agricultural

\footnotetext{
${ }^{\dagger \dagger}$ Available for download at http://lesun.weebly.com/hyperspectral-data-set.html.
} 
Table 8

Parcel map for the first synthetic dataset

\begin{tabular}{|l|l|l|}
\hline 1 & 2 & $\mathbf{3}$ \\
\hline $\mathbf{3}$ & 1 & 2 \\
\hline 2 & $\mathbf{3}$ & 1 \\
\hline
\end{tabular}

fields. This was a case for which non-negative matrix factorization under minimal volume constraint was unique [20, 18, 24]. Only unconstrained non-negative matrix factorization was not unique.

We ran CNN-BTD-Var with $R=3$ and $L=3$; for other algorithms, we used $R=3$. The spectral signatures $\boldsymbol{c}_{r}$ and abundance maps $\boldsymbol{S}_{r}$ are shown in Figures 6 and 7, respectively. The unmixing metrics and computation time are displayed in Table 9.
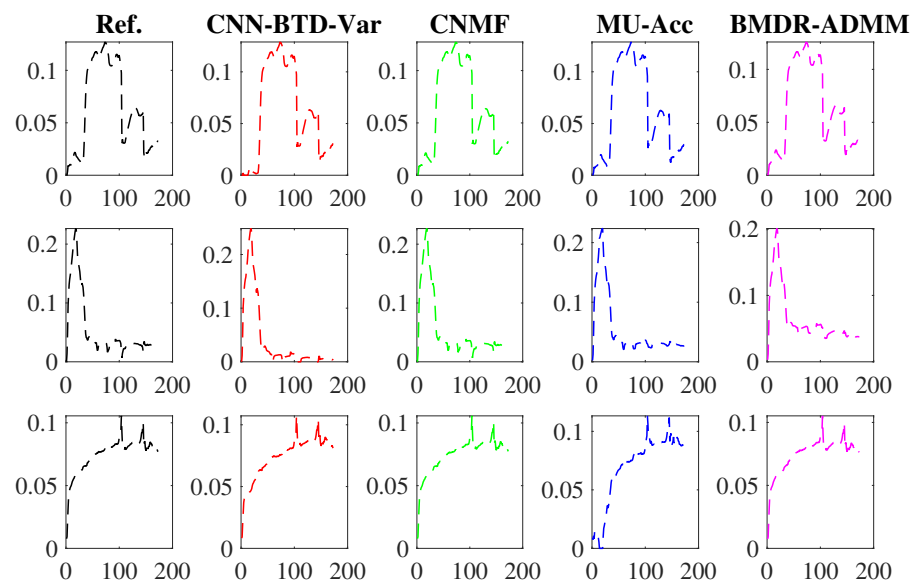

Figure 6. Reference and estimated spectra, synthetic dataset 1

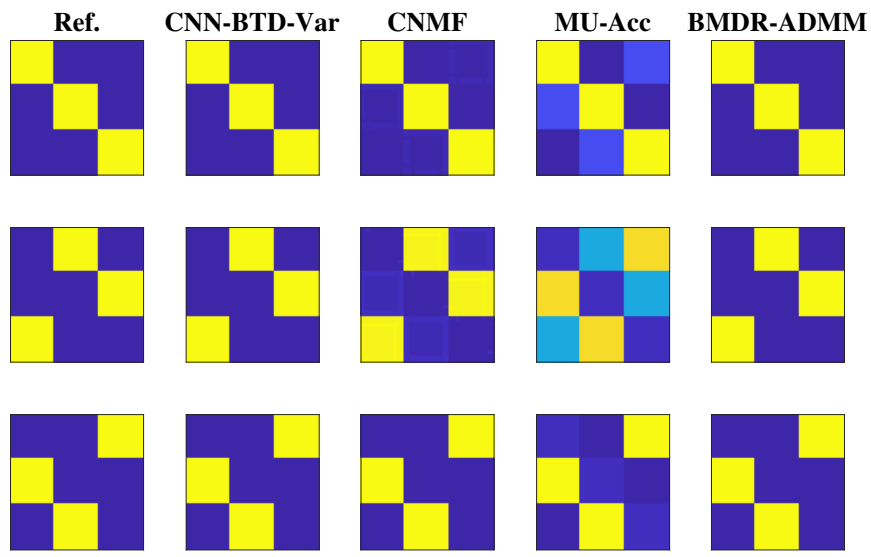

Figure 7. Reference and estimated abundance maps, synthetic dataset 1 
Table 9

Unmixing, synthetic example 1

\begin{tabular}{c|c|c|c|c}
\hline Algorithm & CNN-BTD-Var & CNMF & MU-Acc & BMDR-ADMM \\
\hline SAD & $\mathbf{0 . 0 1 2 3 4 9}$ & 0.300049 & 0.132655 & 0.351785 \\
RMSE & $\mathbf{0 . 1 0 2 4 4 1}$ & 0.274509 & 0.267861 & 0.201845 \\
Time (sec) & $\mathbf{0 . 9 5 8 3 1 1}$ & 1.43277 & 1.73498 & 1.23186
\end{tabular}

We can see that all spectra and abundance maps were recovered accurately by CNNBTD-Var, with visual quality comparable to that of CNMF and BMDR-ADMM. The proposed algorithm gave the best unmixing metrics and computation time. We also see some artifacts in the abundance maps recovered by CNMF. Moreover, MU-Acc did not estimate all abundance maps correctly for this example.

6.2.3. Synthetic example with non-identifiable matrix factorization model. In this second example, we designed an example where the separability (or pure pixel) condition [14, 32] was not fulfilled. This resulted in the traditional non-negative matrix factorization model being non-identifiable. This was in fact a highly mixed situation for which we expected that traditional unmixing algorithms fail at performing unmixing on this dataset. However, the conditions in Theorem 3.1 were satisfied, which made the LL1 factors unique up to permutation and scaling ambiguities. The abundance maps $\boldsymbol{S}_{r}$ were designed as follows:

$$
\boldsymbol{S}_{1}=\frac{1}{12}\left[\begin{array}{lll}
5 & 7 & 6 \\
7 & 3 & 5 \\
3 & 0 & 0
\end{array}\right] \nabla \boldsymbol{H}, \quad \boldsymbol{S}_{2}=\frac{1}{12}\left[\begin{array}{lll}
7 & 5 & 3 \\
0 & 6 & 0 \\
3 & 5 & 7
\end{array}\right] \otimes \boldsymbol{H}, \quad \boldsymbol{S}_{3}=\frac{1}{12}\left[\begin{array}{lll}
0 & 0 & 3 \\
5 & 3 & 7 \\
6 & 7 & 5
\end{array}\right] \nabla \boldsymbol{H},
$$

with $\boldsymbol{H}$ a Gaussian of size $30 \times 30$ with standard deviation $\sigma=5$.

We ran CNN-BTD-Var with $R=3$ and $L=3$; for other algorithms, we used $R=3$. The spectral signatures $\boldsymbol{c}_{r}$ and abundance maps $\boldsymbol{S}_{r}$ are shown in Figures 8 and 9 , respectively. The unmixing metrics are shown in Table 10.
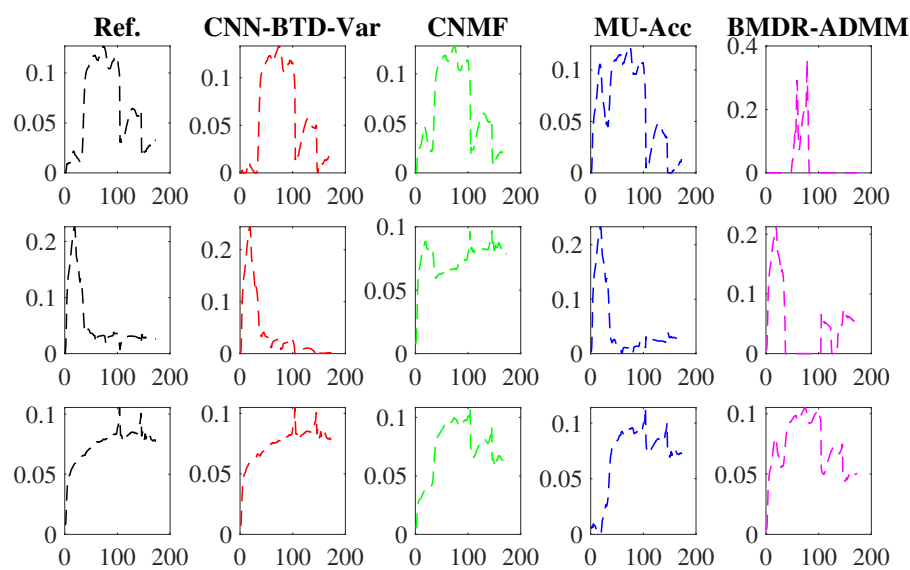

Figure 8. Reference and estimated spectra, synthetic dataset 2

The spectral signatures were best reconstructed by CNN-BTD-Var, although CNMF only reconstructed the first spectrum correcly. Moreover, only CNN-BTD-Var provided reasonable 


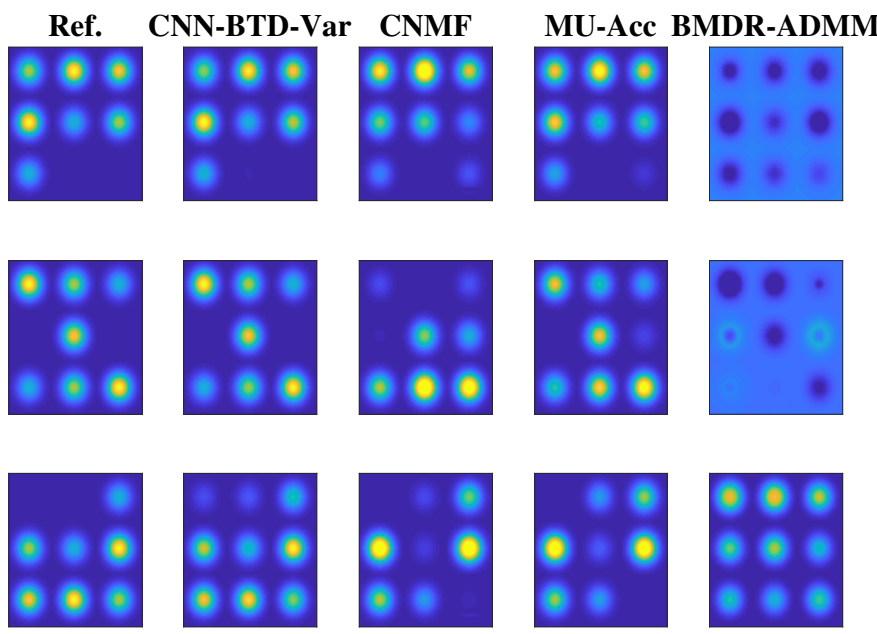

Figure 9. Reference and estimated abundance maps, synthetic dataset 2

Table 10

Unmixing, synthetic example 2

\begin{tabular}{c|c|c|c|c}
\hline Algorithm & CNN-BTD-Var & CNMF & MU-Acc & BMDR-ADMM \\
\hline SAD & $\mathbf{0 . 1 6 0 1 0 9}$ & 0.386413 & 0.355248 & 0.464474 \\
RMSE & $\mathbf{0 . 2 1 0 9 5 2}$ & 0.333595 & 0.253813 & 0.339372 \\
Time (sec) & $\mathbf{0 . 9 7 2 1 5 6}$ & 1.77138 & 1.57192 & 1.20186
\end{tabular}

estimates of the abundance maps, while other algorithms failed. Numerically, the proposed algorithm yielded the best unmixing metrics and computation time.

6.3. Unmixing for real datasets. In this subsection, we assessed unmixing performance of CNN-BTD-Var for real datasets Lake Tahoe and Ivanpah Playa. For the two considered datasets, we followed the same degradation model as in Section 5. For these experiments, the endmembers and abundance maps underlying $\mathcal{Z}$ were unknown: as a result, we chose as reference the spectra and abundance maps selected manually from the SRI $\mathcal{Z}^{\ddagger \ddagger}$. The obtained abundance maps had very close correspondence with visual features in the image. The columns of the abundance maps were rescaled with unit norm for comparison.

6.3.1. Lake Tahoe. We first considered the Lake Tahoe dataset. This dataset was mainly composed of $R=3$ materials: water (lake), soil and vegetation. As a result, we chose $R=3$ and $L=18$ as in the previous subsection. We compared our algorithm with CNMF, MU-Acc and BMDR-ADMM with $R=3$.

On Figures 10 and 11, we plot the estimated spectra and abundance maps.

The proposed approach estimated the spectra accurately. The abundance maps allowed for identification of the areas corresponding to different materials, although with lower resolution than other methods. Additionally, the abundance maps recovered by CNN-BTD-Var seemed to be low-rank. The algorithms CNMF and MU-Acc did not recover the water abundance map correctly, and CNMF did not recover the water spectrum. In Table 11, we show the

\footnotetext{
${ }^{\ddagger \ddagger}$ In real applications, the SRI $\mathcal{Z}$ is unknown. In this paper, we use it as a reference to evaluate the performance of our approach.
} 

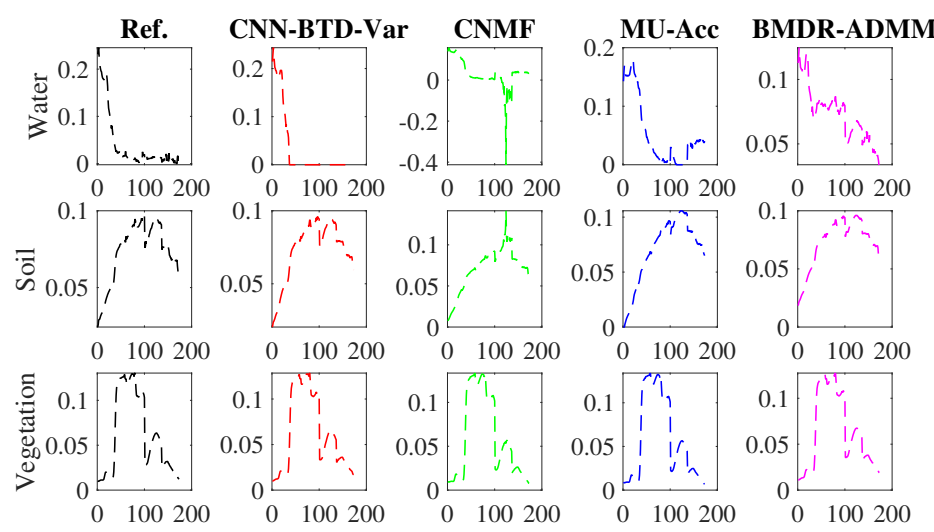

Figure 10. Reference and estimated spectra, Lake Tahoe dataset

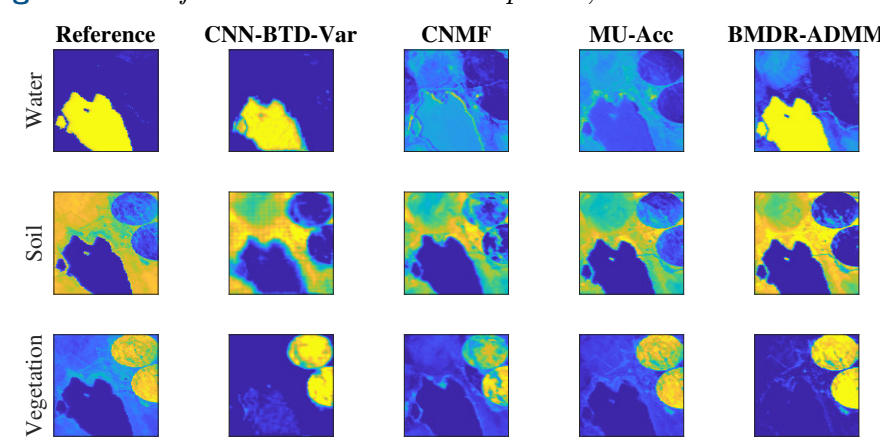

Figure 11. Reference and estimated abundance maps, Lake Tahoe dataset

unmixing and computation time for the considered algorithms. CNN-BTD-Var provided the

Table 11

Unmixing, Lake Tahoe dataset

\begin{tabular}{c|c|c|c|c}
\hline Algorithm & CNN-BTD-Var & CNMF & MU-Acc & BMDR-ADMM \\
\hline SAD & $\mathbf{0 . 0 7 9 4 4 0 6}$ & 0.302813 & 0.1098101 & 0.255009 \\
RMSE & 0.466916 & 0.472743 & 0.637745 & $\mathbf{0 . 3 5 6 7 2 4}$ \\
Time (sec) & $\mathbf{1 . 2 2 9 9 0 6}$ & 1.98253 & 2.0130503 & 1.71438
\end{tabular}

best SAD and computation time, and the second best RMSE after BMDR-ADMM.

Additionally, on Figure 12, we plot the reference and estimated $\widetilde{\boldsymbol{C}}_{M}$ and $\boldsymbol{P}_{3} \boldsymbol{\psi}=\widetilde{\boldsymbol{C}}_{M}-$ $\boldsymbol{P}_{3} \boldsymbol{C}$ obtained from CNN-BTD-Var.

In Figure 12, the water spectrum had high variability for the first MSI spectral band, which corresponds to the blue region. For the vegetation and soil spectra, the largest variability was found at spectral bands corresponding to the green and orange-red wavelengths. Moreover, CNN-BTD-Var recovered the reference $\widetilde{\boldsymbol{C}}_{M}$ and $\boldsymbol{P}_{3} \boldsymbol{\psi}$ with a small discrepancy.

6.3.2. Ivanpah Playa. Next, we considered the Ivanpah Playa dataset. This dataset was composed of $R=4$ materials: solar panels, dark sand, yellow sand and road. We ran CNNBTD-Var with $R=4, L=18$, and compared the results to other baseline algorithms with $R=4$.

In Figures 13 and 14, we plot the reference and estimated spectra and abundance maps. In Table 12, we show the unmixing metrics and computation time. 

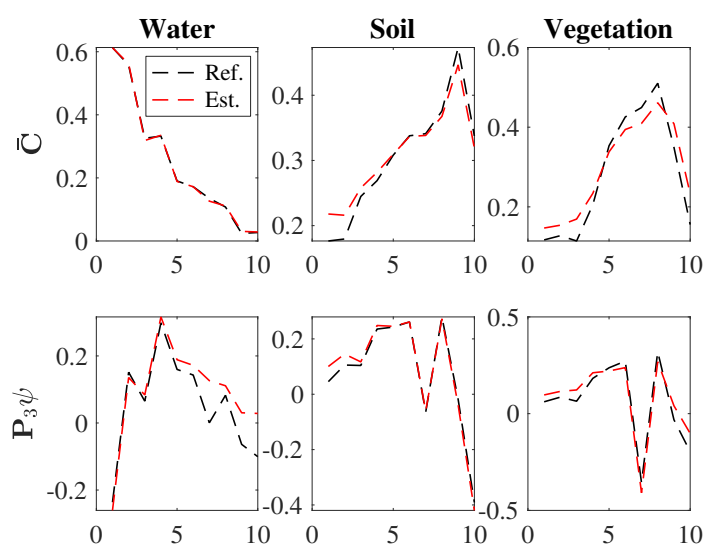

Figure 12. Reference and estimated $\widetilde{\boldsymbol{C}}_{M}$ and $\boldsymbol{P}_{3} \boldsymbol{\psi}$, Lake Tahoe dataset
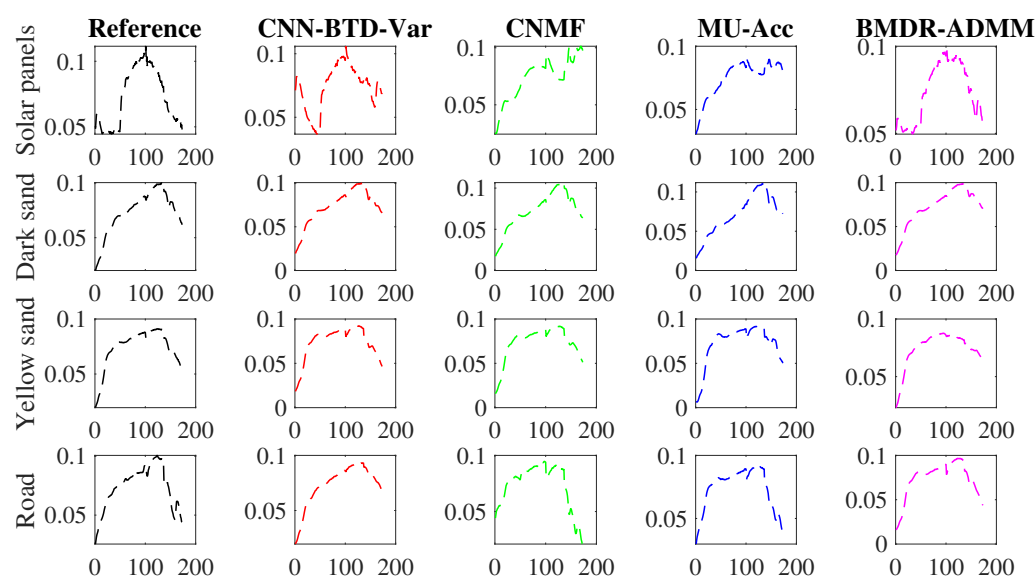

Figure 13. Reference and estimated spectra, Ivanpah Playa dataset

One difficulty for unmixing was the important similarity between the reference spectra (in particular, dark and yellow sand, road materials). This led to almost colinear columns in $\boldsymbol{C}$, which resulted in CNN-BTD-Var giving the worse SAD. This issue was particularly visible in Figure 15 with estimated $\widetilde{\boldsymbol{C}}_{M}$ and $\boldsymbol{P}_{3} \boldsymbol{\psi}$. High variability was found for the red and near-infrared spectral bands for all materials.

Despite this difficulty, CNN-BTD-Var yielded the best SAD and RMSE and recovered the solar panels and road abundance maps best, while the yellow sand map was slightly better for BMDR-ADMM. For this example, the low-rank assumption for abundance maps was reasonable: see that corresponding to solar panels. This assumption allowed for better visual reconstruction of this abundance map. Contrary to other algorithms, all spectra were correctly recovered.

7. Conclusion. In this paper, we proposed new algorithms for solving the HSR problem with variable images, using an LL1-BTD model. First, we showed that in the presence of variability, previous tensor models fail at recovering the SRI, since they do not account for spectral or spatial variability. Our approach allows to recover the SRI accurately for the considered datasets, as well as the degraded variability tensor.

An appropriate choice of ranks also allows our algorithms to estimate underlying spectra 

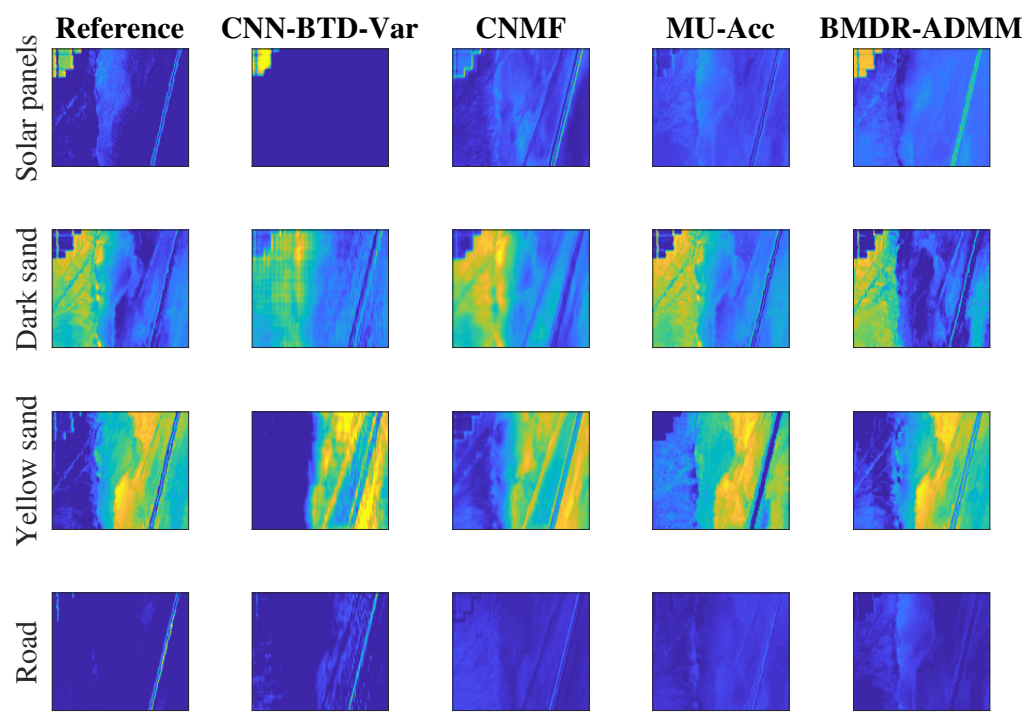

Figure 14. Reference and estimated abundance maps, Ivanpah Playa dataset

Table 12

Unmixing, Ivanpah Playa dataset

\begin{tabular}{c|c|c|c|c}
\hline Algorithm & CNN-BTD-Var & CNMF & MU-Acc & BMDR-ADMM \\
\hline SAD & $\mathbf{0 . 0 9 4 3 4 6}$ & 0.193547 & 0.134738 & 0.113456 \\
RMSE & $\mathbf{0 . 0 0 6 6 9 3}$ & 0.007067 & 0.008188 & 0.007434 \\
Time (sec) & $\mathbf{1 . 3 0 2 5 8}$ & 1.73402 & 1.56564 & 1.679304
\end{tabular}

and abundance maps of the unknown SRI, with performance comparable to those of traditional unmixing algorithms applied on the SRI directly. Non-negativity priors allow the low-rank factors of our model to be interpretable, without having a high negative impact on the computation time.

\section{Appendix A. Unconstrained factor updates in Algorithm 4.1.}

In Algorithm 4.1, the least squares program for $\boldsymbol{A}$ can be seen as a generalized Sylvester equation of the form $\boldsymbol{X}_{1} \boldsymbol{A} \boldsymbol{X}_{2}+\boldsymbol{X}_{3} \boldsymbol{A} \boldsymbol{X}_{4}=\boldsymbol{X}_{5}$, with

$$
\begin{aligned}
\boldsymbol{X}_{1} & =\boldsymbol{P}_{1}^{\top} \boldsymbol{P}_{1}, \quad \boldsymbol{X}_{2}=\left(\boldsymbol{C} \odot_{p} \boldsymbol{P}_{2} \boldsymbol{B}\right)^{\top}\left(\boldsymbol{C} \odot_{p} \boldsymbol{P}_{2} \boldsymbol{B}\right), \quad \boldsymbol{X}_{3}=\lambda \boldsymbol{I}_{I}, \\
\boldsymbol{X}_{4} & =\left(\widetilde{\boldsymbol{C}}_{M} \odot_{p} \boldsymbol{B}\right)^{\top}\left(\widetilde{\boldsymbol{C}}_{M} \odot_{p} \boldsymbol{B}\right), \quad \boldsymbol{X}_{5}=\boldsymbol{P}_{1}^{\top}\left(\boldsymbol{Y}_{H}^{(1)}\right)^{\top}\left(\boldsymbol{C} \odot_{p} \boldsymbol{P}_{2} \boldsymbol{B}\right)+\lambda\left(\boldsymbol{Y}_{M}^{(1)}\right)^{\top}\left(\widetilde{\boldsymbol{C}}_{M} \odot_{p} \boldsymbol{B}\right),
\end{aligned}
$$

and can be solved with efficient solvers. The updates for $\boldsymbol{B}$ and $\boldsymbol{C}$ can be solved similarly.

The pseudo-solution for $\widetilde{\boldsymbol{C}}_{M}$ is expressed as vec $\left\{\widetilde{\boldsymbol{C}}_{M}\right\}=\left(\boldsymbol{X}^{\top} \boldsymbol{X}\right)^{\dagger} \boldsymbol{X}^{\top} \boldsymbol{z}$, with

$$
\begin{aligned}
\boldsymbol{X} & =\left(\sqrt{\lambda} \boldsymbol{S}^{\boldsymbol{\top}} \boldsymbol{S}+\sqrt{\mu} \boldsymbol{I}_{R}\right) \otimes \boldsymbol{I}_{K_{M}}, \\
\boldsymbol{z} & =\operatorname{vec}\left\{\sqrt{\lambda}\left(\boldsymbol{Y}_{M}^{(3)}\right)^{\top} \boldsymbol{S}+\sqrt{\mu}\left(\boldsymbol{P}_{3} \boldsymbol{C}+\boldsymbol{\psi}_{M}\right)\right\} .
\end{aligned}
$$



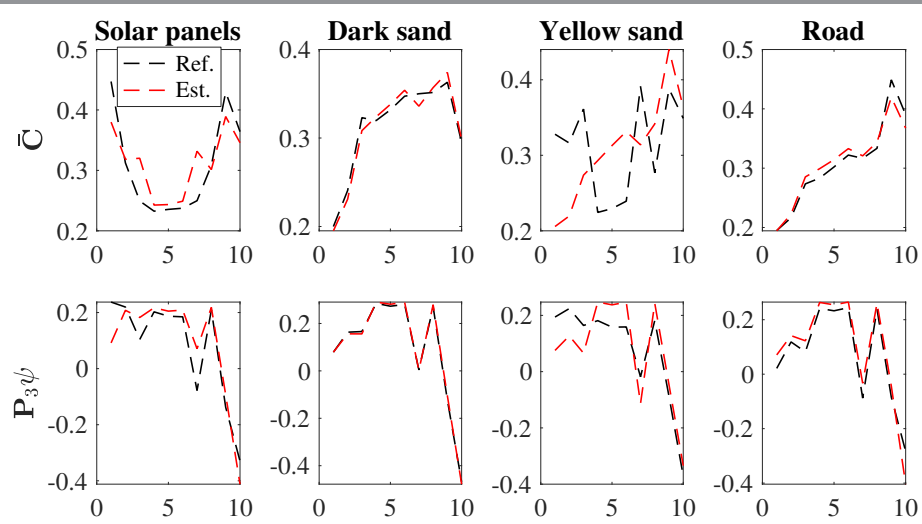

Figure 15. Reference and estimated $\widetilde{\boldsymbol{C}}_{M}$ and $\boldsymbol{P}_{3} \boldsymbol{\psi}$, Ivanpah Playa dataset

Appendix B. Updates in Algorithm 4.2.

Similarly to Algorithm 4.1, the least squares programs for $\boldsymbol{A}$ and $\boldsymbol{B}$ in Algorithm 4.2 can be viewed as generalized Sylvester equations of the form $\boldsymbol{X}_{1} \boldsymbol{A} \boldsymbol{X}_{2}+\boldsymbol{X}_{3} \boldsymbol{A} \boldsymbol{X}_{4}=\boldsymbol{X}_{5}$. For instance, for $\boldsymbol{A}$, we have

$$
\begin{aligned}
& \boldsymbol{X}_{1}=\boldsymbol{P}_{1}^{\top} \boldsymbol{P}_{1}, \quad \boldsymbol{X}_{2}=\left(\boldsymbol{C} \odot_{p} \boldsymbol{P}_{2} \boldsymbol{B}\right)^{\top}\left(\boldsymbol{C} \odot_{p} \boldsymbol{P}_{2} \boldsymbol{B}\right), \quad \boldsymbol{X}_{3}=\boldsymbol{I}_{I}, \\
& \boldsymbol{X}_{4}=\lambda\left(\widetilde{\boldsymbol{C}}_{M} \odot_{p} \boldsymbol{B}\right)^{\top}\left(\widetilde{\boldsymbol{C}}_{M} \odot_{p} \boldsymbol{B}\right)+\gamma \operatorname{Diag}\left\{\boldsymbol{B}_{1}^{\top} \boldsymbol{B}_{1}, \ldots, \boldsymbol{B}_{R}^{\top} \boldsymbol{B}_{R}\right\}, \\
& \boldsymbol{X}_{5}=\boldsymbol{P}_{1}^{\top}\left(\boldsymbol{Y}_{H}^{(1)}\right)^{\top}\left(\boldsymbol{C} \odot_{p} \boldsymbol{P}_{2} \boldsymbol{B}\right)+\lambda\left(\boldsymbol{Y}_{M}^{(1)}\right)^{\top}\left(\widetilde{\boldsymbol{C}}_{M} \odot_{p} \boldsymbol{B}\right)+\gamma\left[\boldsymbol{S}_{1} \boldsymbol{B}_{1}, \ldots, \boldsymbol{S}_{R} \boldsymbol{B}_{R}\right],
\end{aligned}
$$

and likewise for $\boldsymbol{B}$.

At each iteration of the alternating directions method of multipliers scheme, we aim at solving the following equations:

$$
\begin{aligned}
& (\gamma+\rho) \boldsymbol{S}_{r}=\gamma \boldsymbol{A}_{r} \boldsymbol{B}_{r}^{\top}+\rho(\boldsymbol{Z}+\boldsymbol{U}), \\
& \mu \boldsymbol{P}_{3}^{\top} \boldsymbol{P}_{3} \boldsymbol{C}+\boldsymbol{C}\left(\boldsymbol{S}^{\top}\left(\boldsymbol{P}_{2}^{\top} \boldsymbol{P}_{2} \otimes \boldsymbol{P}_{1}^{\top} \boldsymbol{P}_{1}\right) \boldsymbol{S}+\rho \boldsymbol{I}_{R}\right) \\
& =\left(\boldsymbol{Y}_{H}^{(3)}\right)^{\top}\left(\boldsymbol{P}_{2} \otimes \boldsymbol{P}_{1}\right) \boldsymbol{S}+\mu \boldsymbol{P}_{3}^{\top}\left(\boldsymbol{\psi}_{M}-\widetilde{\boldsymbol{C}}_{M}\right)+\rho(\boldsymbol{Z}+\boldsymbol{U}), \\
& \widetilde{\boldsymbol{C}}_{M}\left(\boldsymbol{S}^{\top} \boldsymbol{S}+(\mu+\rho) \boldsymbol{I}_{R}\right)=\left(\boldsymbol{Y}_{M}^{(3)}\right)^{\top} \boldsymbol{S}+\mu\left(\boldsymbol{P}_{3} \boldsymbol{C}+\boldsymbol{\psi}\right)+\rho(\boldsymbol{Z}+\boldsymbol{U}) .
\end{aligned}
$$

For each equation, $\boldsymbol{Z}$ is the projection of the considered variable onto the space of non-negative matrices, and $\boldsymbol{U}$ denotes the dual variable for each subproblem [9]. The scalar $\rho$ controls the convergence speed of the algorithm and is chosen according to [23].

Below, we present the framework for solving (B.2): the updates for $\boldsymbol{S}$ and $\widetilde{\boldsymbol{C}}_{M}$ can be handled in a similar fashion.

Here, the operator $[\cdot]_{+}$zeroes out the negative values of the operand.

\section{Appendix C. Spatial degradation matrices.}

Here, we explain in details how the degradation matrices are constructed. For this appendix, we consider that $\boldsymbol{P}_{1}=\boldsymbol{P}_{2}$. As in previous works, $\boldsymbol{P}_{1}$ is constructed as $\boldsymbol{P}_{1}=\boldsymbol{S}_{1} \boldsymbol{T}_{1}$, where $\boldsymbol{T}_{1}$ is a blurring matrix and $\boldsymbol{S}_{1}$ is a downsampling matrix.

The blurring matrix is constructed from a Gaussian blurring kernel $\phi \in \mathbb{R}^{q \times 1}$ (in our case, 


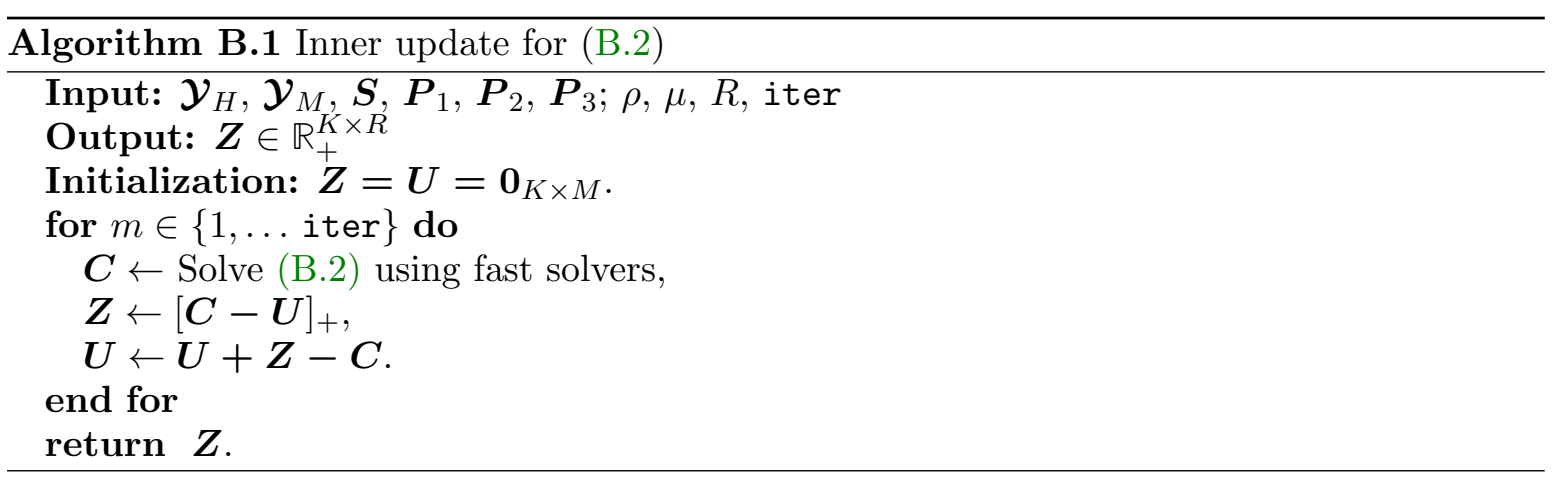

$q=9)$ with a standard deviation $\sigma=\frac{q \sqrt{2 \log 2}}{4}$. For $m \in\{1, \ldots, q\}$ and $m^{\prime}=m-\left\lceil\frac{q}{2}\right\rceil$, we have

$$
\phi(m)=\frac{1}{\sqrt{2 \pi \sigma^{2}}} \exp \left(\frac{-m^{2}}{2 \sigma^{2}}\right)
$$

Thus, $\boldsymbol{T}_{1} \in \mathbb{R}^{I \times I}$ can be expressed as

$$
\boldsymbol{T}_{1}=\left[\begin{array}{cccccc}
\phi\left(\left\lceil\frac{q}{2}\right\rceil\right) & \ldots & \phi(q) & 0 & \ldots & 0 \\
\vdots & \ddots & & \ddots & \ddots & \vdots \\
\phi(1) & & \ddots & & \ddots & 0 \\
0 & \ddots & & \ddots & & \phi(q) \\
\vdots & \ddots & \ddots & & \ddots & \vdots \\
0 & \ldots & 0 & \phi(1) & \ldots & \phi\left(\left\lceil\frac{q}{2}\right\rceil\right)
\end{array}\right] .
$$

The downsampling matrix $\boldsymbol{S}_{1} \in \mathbb{R}^{I_{H} \times I}$, with downsampling ratio $d$, is made of $I_{H}$ independant rows such that for $i \in\left\{1, \ldots, I_{H}\right\},\left(\boldsymbol{S}_{1}\right)_{i, 2+(i-1) d}=1$ and the other coefficients are zeros.

\section{REFERENCES}

[1] B. Aiazzi, L. Alparone, S. Baronti, A. Garzelli, and M. Selva, MTF-tailored multiscale fusion of high-resolution ms and pan imagery, Photogrammetric Eng. and Remote Sens., 72 (2006), pp. 591596.

[2] J. M. Bioucas-Dias And J. P. NAscimento, Hyperspectral subspace identification, IEEE Trans. Geosci. Remote Sens., 46 (2008), pp. 2435-2445.

[3] J. M. Bioucas-Dias, A. Plaza, N. Dobigeon, M. Parente, Q. Du, P. Gader, and J. Chanussot, Hyperspectral unmixing overview: geometrical, statistical, and sparse regression-based approaches, IEEE J. Sel. Topics Appl. Earth Observ. Remote Sens., 5 (2012), pp. 354-379.

[4] R. A. Borsoi, T. Imbiriba, And J. M. Bermudez, Super-resolution for hyperspectral and multispectral image fusion accounting for seasonal spectral variability, IEEE Trans. Image Process., 29 (2020), pp. $116-127$.

[5] R. A. Borsoi, T. Imbiriba, J. M. Bermudez, C. Richard, J. Chanussot, L. Drumetz, J.-Y. Tourneret, And C. ZARe, A. .And JutTen, Spectral variability in hyperspectral data unmixing: A comprehensive review, arXiv preprint arXiv:2001.07307, (2020).

[6] R. A. Borsoi, C. Prévost, K. Usevich, D. Brie, J. M. Bermudez, and C. Richard, Coupled tensor decomposition for hyperspectral and multispectral image fusion with inter-image variability, IEEE J. Sel. Topics Signal Process., (2021). 
[7] M. Bousse, O. Debals, And L. De Lathauwer, A tensor-based method for large-scale blind source separation using segmentation, IEEE Trans. Signal Process., 65 (2016), pp. 346-358.

[8] G. Boutry, M. Elad, G. H. Golub, And P. Milanfar, The generalized eigenvalue problem for nonsquare pencils using a minimal perturbation approach, SIAM J. Matrix Anal. Appl., 27 (2005), pp. 582601 .

[9] S. Boyd, N. PARIKH, AND E. CHU, Distributed optimization and statistical learning via the alternating direction method of multipliers, Now Publishers Inc, 2011.

[10] P. Comon, Tensors: A brief introduction, IEEE Signal Process. Mag., 31 (2014), pp. $44-53$.

[11] L. DE LATHAUWER, Decompositions of a higher-order tensor in block terms-part I: Lemmas for partitioned matrices, SIAM J. Matrix Anal. Appl., 30 (2008), pp. 1022-1032.

[12] L. De Lathauwer, Decompositions of a Higher-Order Tensor in Block Terms-Part II: Definitions and Uniqueness, SIAM J. Matrix Anal. Appl., 30 (2008), pp. 1033-1066.

[13] M. Ding, X. Fu, T.-Z. HuAng, J. WANG, AND X.-L. ZHAO, Hyperspectral super-resolution via interpretable block-term tensor modeling, arXiv e-prints, (2020), arXiv:2006.10248, p. arXiv:2006.10248, https://arxiv.org/abs/2006.10248.

[14] D. Donoho And V. STODDEn, When does non-negative matrix factorization give a correct decomposition into parts?, in Advances in neural information processing systems, 2004, pp. 1141-1148.

[15] A. Eckardt, J. Horack, F. Lehmann, D. Krutz, J. Drescher, M. Whorton, And M. Soutullo, DESIS (DLR earth sensing imaging spectrometer for the ISS-muses platform), in 2015 IEEE IGARSS, 2015, pp. 1457-1459.

[16] M. Elad, P. Milanfar, And G. H. Golub, Shape from moments-an estimation theory perspective, IEEE Trans. Signal Process., 52 (2004), pp. 1814-1829.

[17] I. V. Emelyanova, T. R. McVicar, T. G. Van Niel, L. T. Li, And A. M. Van Dijk, Assessing the accuracy of blending Landsat-MODIS surface reflectances in two landscapes with contrasting spatial and temporal dynamics: A framework for algorithm selection, Remote Sensing of Environment, 133 (2013), pp. 193-209.

[18] X. Fu, K. HuAng, And N. D. Sidiropoulos, On identifiability of nonnegative matrix factorization, IEEE Signal Process. Letters, 25 (2018), pp. 328-332.

[19] X. Fu, W.-K. MA, T.-H. CHAN, AND J. M. BiouCAS-DiAs, Self-dictionary sparse regression for hyperspectral unmixing: Greedy pursuit and pure pixel search are related, IEEE J. Sel. Topics Signal Process., 9 (2015), pp. 1128-1141.

[20] X. Fu, W.-K. MA, K. HuAng, And N. D. Sidiropoulos, Blind separation of quasi-stationary sources: Exploiting convex geometry in covariance domain, IEEE Trans. Signal Process., 63 (2015), pp. 23062320 .

[21] N. Gillis AND F. GLineur, Accelerated multiplicative updates and hierarchical als algorithms for nonnegative matrix factorization, Neural computation, 24 (2012), pp. 1085-1105.

[22] T. Hilker, M. A. Wulder, N. C. Coops, J. Linke, G. McDermid, J. G. Masek, F. Gao, and J. C. White, A new data fusion model for high spatial-and temporal-resolution mapping of forest disturbance based on landsat and MODIS, Remote Sensing of Environment, 113 (2009), pp. 16131627.

[23] K. Huang, N. D. Sidiropoulos, and A. P. Liavas, A flexible and efficient algorithmic framework for constrained matrix and tensor factorization, IEEE Trans. Signal Process., 64 (2016), pp. 5052-5065.

[24] K. Huang, N. D. Sidiropoulos, And A. Swami, Non-negative matrix factorization revisited: Uniqueness and algorithm for symmetric decomposition, IEEE Trans. Signal Process., 62 (2013), pp. 211-224.

[25] T. Imbiriba, R. A. Borsoi, And J. M. Bermudez, Generalized linear mixing model accounting for endmember variability, in 2018 IEEE ICASSP, 2018, pp. 1862-1866.

[26] M.-D. Iordache, J. M. Bioucas-Dias, and A. Plaza, Sparse unmixing of hyperspectral data, IEEE Trans. on Geosci. Remote Sens., 49 (2011), pp. 2014-2039.

[27] C. I. Kanatsoulis, X. Fu, N. D. Sidiropoulos, and W.-K. Ma, Hyperspectral Super-Resolution: A Coupled Tensor Factorization Approach, IEEE Trans. Signal Process., 66 (2018), pp. 6503-6517.

[28] C. I. Kanatsoulis, X. Fu, N. D. Sidiropoulos, and W.-K. Ma, Hyperspectral Super-Resolution: Combining Low Rank Tensor and Matrix Structure, in 2018 IEEE ICIP, Oct. 2018, pp. 3318-3322, https://doi.org/10.1109/ICIP.2018.8451733.

[29] H. Kaufmann, K. Segl, S. Chabrillat, S. Hofer, T. Stuffler, A. Mueller, R. Richter, G. SCHREIER, R. HAYDN, AND H. BACH, EnMAP a hyperspectral sensor for environmental mapping and analysis, in 2006 IEEE IGARSS, 2006, pp. 1617-1619.

[30] N. Keshava and J. F. Mustard, Spectral unmixing, IEEE Signal Processing Magazine, 19 (2002), pp. $44-57$. 
[31] T. G. Kolda And B. W. Bader, Tensor Decompositions and Applications, SIAM Review, 51 (2009), pp. 455-500.

[32] H. Laurberg, M. G. Christensen, M. D. Plumbley, L. K. Hansen, and S. H. Jensen, Theorems on positive data: On the uniqueness of NMF, Computational intelligence and neuroscience, 2008 (2008).

[33] Q. LI, W.-K. MA, AND Q. WU, Hyperspectral super-resolution: Exact recovery in polynomial time, in 2018 IEEE SSP, IEEE, 2018, pp. 378-382.

[34] H. LiU, R. WU, AND W.-K. MA, Is there any recovery guarantee with coupled structured matrix factorization for hyperspectral super-resolution?, in 2019 IEEE CAMSAP, IEEE, 2019, pp. 480-484.

[35] J. NASCimento AND J. Dias, Vertex component analysis: A fast algorithm to unmix hyperspectral data, IEEE Geosci. Remote Sens. Lett., 43 (2005), pp. 898-910.

[36] L. Nus, Méthodes rapides de traitement d'images hyperspectrales. Application à la caractérisation en temps réel du matériau bois, $\mathrm{PhD}$ Thesis, University of Lorraine, France, 2019.

[37] M. PARENTE AND A. PlazA, Survey of geometric and statistical unmixing algorithms for hyperspectral images, in 2nd IEEE Workshop on Hyperspectral Image and Signal Process.: Evolution in Remote Sens., 2010, pp. 1-4.

[38] C. Prévost, K. Usevich, P. Comon, and D. Brie, Hyperspectral Super-Resolution with Coupled Tucker Approximation: Identifiability and SVD-based algorithms, IEEE Trans. Signal Process., 68 (2020), pp. 931-946.

[39] Y. QIAN, F. Xiong, S. Zeng, J. Zhou, AND Y. TANG, Matrix-vector nonnegative tensor factorization for blind unmixing of hyperspectral imagery, IEEE Trans. Geosci. Remote Sens., 55 (2016), pp. 1776-1792.

[40] R. E. Roger And J. F. Arnold, Reliably estimating the noise in AVIRIS hyperspectral images, International Journal of Remote Sensing, 17 (1996), pp. 1951-1962.

[41] G. A. Shaw And H. K. Burke, Spectral imaging for remote sensing, Lincoln laboratory journal, 14 (2003), pp. 3-28.

[42] M. Simoes, J. M. Bioucas-Dias, L. B. Almeida, And J. Chanussot, A convex formulation for hyperspectral image superresolution via subspace-based regularization, IEEE Trans. Geosci. Remote Sens., 53 (2015), pp. 3373-3388.

[43] V. Simoncini, Computational methods for linear matrix equations, SIAM Review, 58 (2016), pp. $377-441$.

[44] B. Somers, G. P. Asner, L. Tits, and P. Coppin, Endmember variability in spectral mixture analysis: A review, Remote Sensing of Environment, 115 (2011), pp. 1603-1616.

[45] M. SøREnSEn AND L. De LATHAUwer, Fiber sampling approach to canonical polyadic decomposition and application to tensor completion, SIAM J. Matrix Anal. Appl., 40 (2019), pp. 888-917.

[46] N. Vervliet, O. Debals, L. Sorber, M. V. Barel, and L. D. Lathauwer, Tensorlab 3.0, Available online, 2016.

[47] L. Wald, T. RANChin, AND M. Mangolini, Fusion of satellite images of different spatial resolutions: Assessing the quality of resulting images, Photogrammetric Eng. and Remote Sens., 63 (1997), pp. 691699.

[48] Q. Wei, J. M. Biouchs-Dias, N. Dobigeon, And J.-Y. Tourneret, Multiband image fusion based on spectral unmixing, IEEE Trans. Geosci. Remote Sens., 54 (2016), pp. 7236-7249.

[49] Q. Wei, N. Dobigeon, And J.-Y. Tourneret, Fast fusion of multi-band images based on solving a Sylvester equation, IEEE Trans. Image Process., 24 (2015), pp. 4109-4121.

[50] N. Yokoya, C. Grohnfeldt, And J. Chanussot, Hyperspectral and multispectral data fusion: A comparative review of the recent literature, IEEE Trans. Geosci. Remote Sens., 5 (2017), pp. 29-56.

[51] N. YokOYA, T. YAIRI, AND A. IwASAKI, Coupled Nonnegative Matrix Factorization Unmixing for Hyperspectral and Multispectral Data Fusion, IEEE Trans. Geosci. Remote Sens., 50 (2012), pp. 528-537, https://doi.org/10.1109/TGRS.2011.2161320.

[52] A. ZARE AND K. C. Ho, Endmember variability in hyperspectral analysis: Addressing spectral variability during spectral unmixing, IEEE Signal Process. Mag., 31 (2013), pp. 95-104.

[53] G. Zhang, X. Fu, K. HuAng, AND J. WANG, Hyperspectral super-resolution: A coupled nonnegative block-term tensor decomposition approach, in 2019 IEEE CAMSAP, 2019. Guadeloupe, West Indies.

[54] G. Zhang, X. Fu, J. WAng, X.-L. Zhao, And M. Hong, Spectrum cartography via coupled block-term tensor decomposition, IEEE Trans. Signal Process., (2020). 